\title{
A PL GEOMETRIC STUDY OF ALGEBRAIC $K$-THEORY
}

\author{
BIZHONG HU
}

\begin{abstract}
This paper manages to apply the Farrell-Jones theory on algebraic $K$-groups of closed negatively curved riemannian manifolds to Gromov's hyperbolic group theory. The paper reaches the conclusion that for any finite polyhedron $K$ with negative curvature, $\mathrm{Wh}\left(\pi_{1} K\right)=0$.
\end{abstract}

\section{INTRODUCTION}

This paper shall prove the following result: For any finite polyhedron $K$ with negative curvature, $\mathrm{Wh}\left(\pi_{1} K \times \mathbb{Z}^{i}\right)=0, i \geq 0$.

Our study originates from an attempt to carry a very successful strategy created by F. T. Farrell and L. E. Jones in [FJ1] into M. Gromov's theory of hyperbolic groups [G1], to "say something about the Whitehead groups of hyperbolic groups."

To describe the theory of Farrell-Jones in [FJ1], first recall the $s$-cobordism theorem: Let $W$ be an $h$-cobordism over $M^{n}, n \geq 5$. Then $W$ is a product if and only if $\tau(W) \in \mathrm{Wh}\left(\pi_{1} M\right)$ vanishes; any element of $\mathrm{Wh}\left(\pi_{1} M\right)$ is the Whitehead torsion of an $h$-cobordism over $M$. Next there is the control theory: This theory measures an $h$-cobordism by a real number, the diameter, and tells that the $h$-cobordism is a product when the diameter is small enough. Along these lines, Farrell and Jones consider any compact riemannian manifold $M$ with $K<0$. They make use of the geodesic flow which is Anosov to change $h$-cobordisms over $M$ and apply their foliated control result to obtain the following.

Theorem (Farrell-Jones, [FJ1]). For any compact riemannian manifold $M$ with $K<0, \operatorname{Wh}\left(\pi_{1} M \times \mathbb{Z}^{i}\right)=0, i \geq 0$. (It is now formally announced in [FJ3] that $\mathrm{Wh}\left(\pi_{1} M \times \mathbb{Z}^{i}\right)=0$ even when $K \leq 0$.)

The concept of a polyhedron with $K \leq 0$ is defined and studied in [G1]. Note that fundamental groups of finite polyhedra with negative curvature constitute a major source for hyperbolic groups.

Now we give a description of our approach. Let $M$ be a PL manifold with $K \leq 0$. Define the ray space $R$ to be the collection of all geodesic rays. $R \rightarrow M$ is a fiber bundle. It is used to asymptotically lift an $h$-cobordism from $M$ to $R$. Define the geodesic space $G$ to be the collection of all geodesics. $G$, rather than $R$, is the geodesic flow, but $G \rightarrow M$ is not a fiber bundle. Fortunately,

Received by the editors September 11, 1990.

1980 Mathematics Subject Classification (1985 Revision). Primary 18F25; Secondary 57Q10. 
the canonical map $G \rightarrow R$ can be approximated by homeomorphisms, due to a result of M. Davis and T. Januszkiewicz in [DJ] and the theory of cell-like maps of L. Siebenmann. So we can take an $h$-cobordism from $R$ to $G$ and change it by the time action of $G$. The metric of $G$ is from its universal covering $G(X)$. At $G(X)$, use the following metric

$$
d(\alpha, \beta)=\int_{-\infty}^{+\infty} d[\alpha(t), \beta(t)] \cdot e^{-|t|} d t .
$$

And there is a relating concept, the weakly Anosov flow. Next we face a foliation $G$ that is merely topological. To treat $G$ we make use of the topological transversality of Kirby-Siebenmann-Quinn [Q2] to establish a concept called stratified decomposition for topological manifolds and use it to obtain a $C^{0}$ foliated control result, the topological version of a $C^{\infty}$ foliated control result of Farrell-Jones. Then we can show that for any compact PL manifold $M$ with $K<0, \mathrm{Wh}\left(\pi_{1} M \times \mathbb{Z}^{i}\right)=0, i \geq 0$. Finally we apply the concept of hyperbolization of Gromov and F. Waldhausen's result on Whiteheads groups of free products with amalgamations to extend the result for any finite polyhedron with curvature $<0$.

I would like to sincerely thank Lowell Jones, who introduced me to their theory and brought me key documents.

\section{PL GEOMETRY AND GEODESIC RAMIFICATION}

1.1. Notations. $\mathbb{R}^{n}, \mathbb{H}^{n, \varepsilon}, \varepsilon<0$, and $S^{n, \varepsilon}, \varepsilon>0$, is, respectively, the $n$ dimensional simply connected riemannian space with curvature $0, \varepsilon<0$ and $\varepsilon>0$.

1.2. Definition. Fix $\varepsilon \in \mathbb{R}$. Let $K$ be a finite dimensional simplicial complex. For each simplex $\Delta^{k} \subset K$, we can realize it in the $k$-dimensional space form of curvature $\varepsilon$ and give it the resulting geometry. We can do so in such a way that all geometric simplices in $K$ glue together to give a PL geometry to $K$ : For any PL curve $\alpha=\alpha_{1} \cup \cdots \cup \alpha_{k}$, where each $\alpha_{i}, 1 \leq i \leq k$, is a line segment in a simplex in $K$, define its length as $L(\alpha)=L\left(\alpha_{1}\right)+\cdots+L\left(\alpha_{k}\right)$. Distance is minimum of lengths. A geodesic segment is a local isometry $[a, b] \rightarrow K . K$ is called a PL $\varepsilon$-space.

We mention that for any two points in $K$ there exists a PL curve with minimum length, any line segment in a simplex is a geodesic of $K$ and that a line segment as a geodesic does not ramify at interior points. For other discussions, see [DJ, §2], [G1, §1.4], and [Mo].

Let $n$ be an integer and $\varepsilon \leq 0$. Denote $r=\left(e^{\sqrt{|\varepsilon|}}-e^{-\sqrt{|\varepsilon|}}\right) / 2 \sqrt{|\varepsilon|}$. Recall that in $\mathbb{H}^{n, \varepsilon}$, a unit sphere with its induced metric is isometric to $S^{n-1}(r)$, i.e., $S^{n-1,1 / r^{2}}$. Let $K$ be a PL $\varepsilon$-space. Consider any $x \in K$. Take a simplex $\Delta^{k}$, $x \in \Delta^{k}$. We know that $\Delta^{k}$ is realized in $\mathbb{H}^{k, \varepsilon}$. Take a face $\Delta^{l} \subset \Delta^{k}, x \notin \Delta^{l}$. Consider the collection of unit tangent vectors at $x$ in $\mathbb{H}^{k, \varepsilon}$ whose geodesic rays reach $\Delta^{l}$. This is an $l$-simplex in $S^{l, 1 / r^{2}}$. All these glue together to form a PL $1 / r^{2}$-space, $\operatorname{link}(x)$, called the link at $x$. The following fact appears in $[\mathrm{G} 1, \S 4.2]$.

1.3. Proposition. Let $K$ be a $P L \varepsilon$-space, $\varepsilon \leq 0$. Then the following are equivalent. 


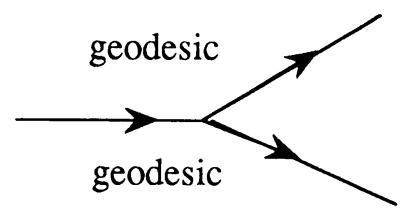

FIGURE 1

(1) $K \leq \varepsilon$. Its meaning will be cited in $\S 2$.

(2) For any $x \in K, \operatorname{link}(x)$ is large, that is, for any $a, b \in \operatorname{link}(x)$, $d(a, b)<r(\varepsilon) \cdot \pi, a$ and $b$ are joined by a unique geodesic in $\operatorname{link}(x)$.

For a triangulated surface with a PL $\varepsilon$-geometry, $\varepsilon \leq 0$, curvture $\leq \varepsilon$ if and only if at any vertex the sum of all angles satisfies $\sum \theta \geq 2 \pi$.

In a PL space with curvature $\leq \varepsilon \leq 0$, one faces the phenomenon shown in Figure 1.

But, using a concept called infinitesimal shadow together with theories on near homeomorphisms in topology, [DJ, §2-§3] proves the following results.

1.4. Theorem. Suppose that $X$ is a simply connected PL n-manifold with a PL geometry such that $K \leq \varepsilon \leq 0$.

(1) For $x \in X, r>0, E_{x}(r)$ is homeomorphic to the usual $E^{n}$.

(2) For $x \in X, 0<r<R$, the canonical map $S_{x}(R) \rightarrow S_{x}(r)$ can be approximated by homeomorphisms.

\section{Spaces With $K \leq 0$}

We now carry out a general study aimed at complete PL spaces with $K \leq$ $\varepsilon \leq 0$. For the following definitions, refer to [G1, §4] and [G2].

\subsection{Definitions.}

Let $M$ be a topological space. Suppose for each curve $\alpha$ in $M$ there is number $L(\alpha)$ such that $L(\alpha) \geq 0, L(\alpha)=0$ only when $\alpha$ is a point, $L(\alpha)=$ $L\left(\alpha^{-1}\right)$ and $L(\alpha * \beta)=L(\alpha)+L(\beta)$. Then call $M$ with $L$ a length space. Define distance as the minimum of lengths to get a metric for $M$. A geodesic is a local isometry $\mathbb{R} \rightarrow M$. Always assume that a geodesic segment can be extended and that closed balls are compact.

A triangle in a length space is composed of three points and three geodesic segments connecting them. We express a triangle $\Delta=\left(x_{1}, x_{2}, x_{3}\right)$, or, more precisely $\Delta=\left(x_{1}, x_{2}, x_{3}, \alpha_{1}, \alpha_{2}, \alpha_{3}\right)$, where $\left(x_{1}, x_{2}, x_{3}\right)$ are three points, $\left(\alpha_{1}, \alpha_{2}, \alpha_{3}\right)$ are unoriented geodesic segments connecting $x_{2}$ and $x_{3}, x_{3}$ and $x_{1}, x_{1}$ and $x_{2}$. Denote $|\Delta|=\left(L\left(\alpha_{1}\right), L\left(\alpha_{2}\right), L\left(\alpha_{3}\right)\right)$. Two triangles $\Delta$ and $\widetilde{\Delta}$ in two length spaces are equivalent if $|\Delta|=|\tilde{\Delta}|$.

Suppose $\Delta$ and $\widetilde{\Delta}$ are equivalent triangles in length spaces $M$ and $N$. We say that $\Delta$ is thinner than $\widetilde{\Delta}$ if the following is always true. (See Figure 2.)

Suppose that $M$ is a length space such that any small triangle in $M$ is thinner than its equivalent triangle in $\mathbb{H}^{2, \varepsilon}, \varepsilon \leq 0$, then $M$ is said to be a space with $K \leq \varepsilon$. Call a simply connected space with $K \leq 0$ a Hadamard space. 


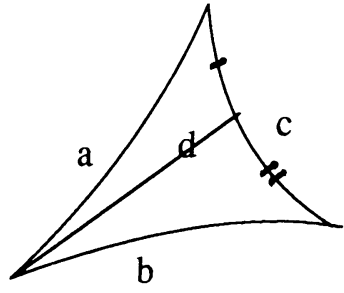

$\Delta$

$\mathrm{d} \leq \widetilde{\mathrm{d}}$

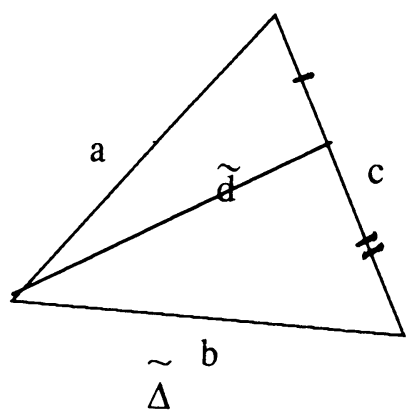

FIGURE 2

\subsection{The ideal boundary.}

2.2.1. Proposition. Let $X$ be a Hadamard space. Then for any two geodesics $\alpha(t)$ and $\beta(t), f(t)=d[\alpha(t), \beta(t)], t \in \mathbb{R}$, is convex. See $[\mathrm{G} 1, \S 4]$.

For a Hadamard space $X$, any geodesic $\mathbb{R} \rightarrow X$ is an isometry, two points can be joined by a unique geodesic segment. These are seen by 2.2.1. Two geodesics $\alpha(t)$ and $\beta(t)$ are said to be asymptotic if $d[\alpha(t), \beta(t)], t \geq 0$, is bounded.

2.2.2. Proposition. Let $X$ be a Hadamard space $x \in X, \alpha(t), t \geq 0, a$ geodesic ray. Then there is unique geodesic ray from $x$ which is asymptotic to $\alpha$.

Proof. Uniqueness is seen by 2.2.1. Consider for any $T \geq 0$ the geodesic segment $\beta_{T}$. (See Figure 3.)

Take any sequence $\left(T_{i}\right) \rightarrow+\infty$. Since $S_{x}(1)$ is compact, there is a subsequence $\left(T_{i_{1}}\right) \subset\left(T_{i}\right)$ such that $\beta_{T_{i_{1}}}(1)$ is convergent. In the same way, there is a subsequence $\left(T_{i_{2}}\right) \subset\left(T_{i_{1}}\right)$ such that $\beta_{T_{i_{2}}}(2)$ is convergent and so on. Then we have subsequence $\left(T_{i_{1}}\right) \subset\left(T_{i}\right)$ such that $\beta_{T_{i_{i}}}(k)$ is convergent for any $k=1,2,3, \ldots$. Then $\beta_{T_{i_{i}}} \rightarrow \beta$ pointwise. Note that if a collection of geodesic segments converges pointwise to a curve, that curve must be a geodesic segment. So $\beta(t), t \geq 0$, is a geodesic ray. (See Figure 4.)

By 2.2.1, $d_{i} \leq L, i \rightarrow+\infty: d[\alpha(t), \beta(t)] \leq L, t \geq 0$. In conclusion $\lim _{T \rightarrow+\infty} \beta_{T}=\beta$ pointwise and $\beta$ is asymptotic to $\alpha$. This completes the proof.

Consider a Hadamard space $X$. An asymptotic relation is an equivalence relation for geodesic rays. The collection of asymptotic classes is denoted $\partial X$. Consider any $z \in \partial X$. Taking any $x \in X$, and any $r>0, y \in S_{x}(r)$ is the point between $x$ and $z$. Take any neighborhood $S$ of $y$ in $S_{x}(r)$. Consider

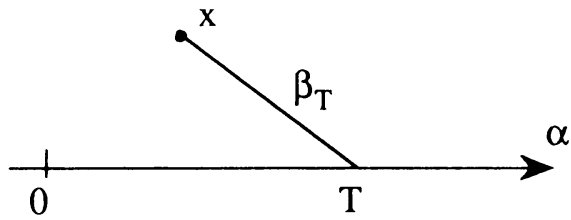

FIGURE 3 




FIGURE 4

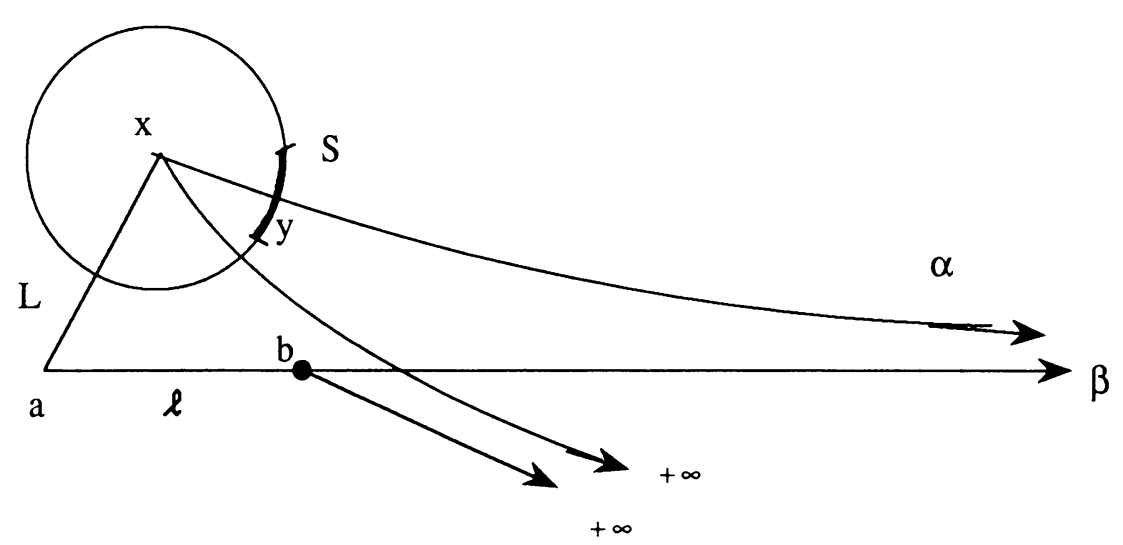

FIGURE 5

all geodesic rays from $x$ though $S$ and we have $\partial \subset \partial X$. Take any set $A$ of $\partial X$ containing $\partial$. Define $\Sigma_{z}=\{A\}$. If the following are true for $\left\{\Sigma_{z}, z \in \partial X\right\}$, then we have unique topology for $\partial X$ such that $\left\{\Sigma_{z}, z \in \partial X\right\}$ are neighborhoods.

(1) $A \in \Sigma_{z} \Rightarrow z \in A$.

(2) $A, B \in \Sigma_{z} \Rightarrow A \cap B \in \Sigma_{z}$.

(3) $A \subset B, A \in \Sigma_{z} \Rightarrow B \in \Sigma_{z}$.

(4) $\forall A \in \Sigma_{z}, \exists U \subset A, U \in \Sigma_{z}$ such that $\forall \omega \in U, U \in \Sigma_{w}$.

Note that the second condition needs verification. The following lemma does the job.

Let $X$ be a Hadamard space, $z \in \partial X, x \in X, \alpha$ is the geodesic ray from $x$ to $z X, r>0, y \in S_{x}(r)$ is the point between $x$ and $z, S$ is a neighborhood of $y$ in $S_{x}(r)$ and $\partial \subset \partial X$ is the collection of geodesic rays from $x$ through $S$. And the same is true for $\tilde{x}, \tilde{\alpha}, \tilde{r}, \tilde{y}, \tilde{S}, \tilde{\partial}$. Take any $a \in X ; \beta$ is the geodesic ray from $a$ to $z$. For $l>0, b$ is the point in $S_{l}(a)$ between $a$ and $z$. The collection of points in $S_{l}(a)$ that are between $a$ and $\partial \cap \tilde{\partial}$ denoted $N$.

2.2.3. Lemma. There is $l$ such that $N$ is a neighborhood of $b$ in $S_{a}(l)$.

Proof. Suppose no $l$ can be found. Then we may suppose that for each $l>0$, $\alpha_{i}$ are geodesic rays from $x$ through $S_{x}(r)-S, \beta_{i}$ are geodesic rays from $a$ through $b_{i} \in S_{a}(l), b_{i} \rightarrow b, \alpha_{i}$ and $\beta_{i}$ are asymptotic. Since spheres are compact, we may well suppose that $\alpha_{i} \rightarrow \alpha_{+\infty}, \beta_{i} \rightarrow \beta_{+\infty}$ pointwise. Then $\alpha_{+\infty}$ is a geodesic ray from $x$ through $S_{x}(r)-S^{0}, \beta_{+\infty}$ is a geodesic ray from $a$ through $b, \alpha_{+\infty}$ and $\beta_{+\beta}$ are asymptotic. (See Figure 5.) 


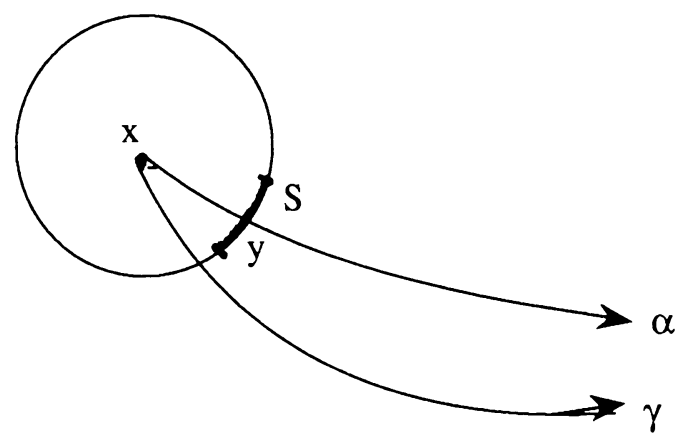

FIGURE 6

In particular $d\left[\alpha_{+\infty}(t), \beta(t)\right] \leq L, 0 \leq t \leq l$. Choose $l_{j} \rightarrow+\infty$ and consider $\alpha_{+\infty}, j$. Again we may well suppose that $\alpha_{+\infty}, j \rightarrow \gamma$ pointwise. Then $\gamma$ is a geodesic ray from $x$ through $S_{x}(r)-S^{0}$. And $d[\gamma(t), \beta(t)] \leq L$, $t \geq 0$. Since $\alpha$ and $\beta$ are asymptotic, $\gamma$ and $\alpha$ are asymptotic. (See Figure 6.)

This is impossible by 2.2 .1 .

2.2.4. Proposition. Suppose $X$ is a Hadamard space.

(1) For geodesic rays $\alpha_{i}$ and $\alpha, \alpha_{i} \rightarrow \alpha$ pointwise if and only if $\alpha_{i}(0) \rightarrow \alpha(0)$ and $\alpha_{i}(+\infty) \rightarrow \alpha(+\infty)$.

(2) For geodesics $\alpha_{i}$ and $\alpha, \alpha_{i} \rightarrow \alpha$ pointwise if and only if $\alpha_{i}(0) \rightarrow \alpha(0)$, $\alpha_{i}(+\infty) \rightarrow \alpha(+\infty), \alpha_{i}(-\infty) \rightarrow \alpha(-\infty)$.

(3) $\partial X=\lim _{\longleftarrow} S_{x}(r)$.

2.3. We now list some conclusions from [Hu, 4.2-4.5], where details can be found.

2.3.1. Proposition. Suppose $M$ is a compact space with $K \leq 0$ such that there is no totally geodesic $S^{1}(r) \times[0, l]$ immersed in $M$. Then for any constant $C$, the following is a finite set:

$$
\{\text { periodic geodesics with periods } \leq C\} .
$$

Suppose $X$ is a Hadamard space. $f(t)=\left(e^{t}+e^{-t}\right) / 2$. Consider $X \times \mathbb{R}$. For any curve in $X \times \mathbb{R}, \alpha(t) \times \beta(t), 0 \leq t \leq 1$, define its length by the following formula, which can be understood as the maximum of discrete sums.

$$
L=\int_{0}^{1} \sqrt{\{f[\beta(t)]\}^{2} \cdot\left|\alpha^{\prime}(t)\right|^{2}+\left|\beta^{\prime}(t)\right|^{2}} d t .
$$

\subsubsection{Proposition.}

(1) $X \times \mathbb{R}$ is a Hadamard space.

(2) $X \times 0 \subset X \times \mathbb{R}$ is totally geodesic.

(3) $x \times \mathbb{R}$ is totally geodesic, $x \in X$.

(4) For any isometry $\sigma, \sigma \times$ Id is isometry.

(5) If $\alpha \times \beta$ is geodesic, Image $\alpha \subset X$ must be geodesic.

(6) For any geodesic $\alpha$ of $X, \alpha \times \mathbb{R}$ is totally geodesic and is isometric to the standard $\mathbb{H}^{2}$. 


\section{THE SPACE OF GEODESICS}

This section lists some conclusions from [Hu, §5], where details can be found.

3.1. Definition. Suppose $X$ is a Hadamard space. Define $G(X)=\{\mathbb{R} \rightarrow X$ isometry\}. The following metric to $G(X)$ appears in [G1, 8.3].

$$
d(\alpha, \beta)=\int_{-\infty}^{+\infty} d[\alpha(t), \beta(t)] \cdot e^{-|t|} d t .
$$

We will call this the geodesic metric. $\mathbb{R}$ naturally acts on $G(X)$, defining the geodesic flow. For a geodesic $\alpha(t), \tau \in \mathbb{R}$, the action produces a geodesic $\alpha(t+\tau)$. The distance between these two geodesics is $2|\tau|$.

3.2. Theorem. Let $X$ be a Hadamard space and $G(X)$ the space of geodesics. Then for any two sequences of geodesics $\alpha_{i}$ and $\beta_{i}, d\left(\alpha_{i}, \beta_{i}\right) \rightarrow 0$ if and only if $d\left[\alpha_{i}(t), \beta_{i}(t)\right] \rightarrow 0, \forall t \in \mathbb{R}$.

3.3. Definition. Suppose $M$ is a space with $K \leq 0$. Define $G(M)=\{\mathbb{R} \rightarrow M$ local isometry\}. Suppose $X \rightarrow X / \Gamma=M$ is the universal covering. $G(X) \rightarrow$ $G(X) / \Gamma=G(M)$ is a universal covering. From the geodesic metric of $G(X)$ we get the geodesic metric of $G(M) . \mathbb{R}$ acts naturally on $G(M)$, defining the geodesic flow.

3.4. Corollary. Suppose $M$ is a space with $K \leq 0$ and $X$ is the universal covering. $\alpha(t), \beta(t)$ are geodesics in $M$ and $\tilde{\alpha}(t), \tilde{\beta}(t)$ are liftings of $\alpha(t)$, $\beta(t)$. Then $d(\alpha, \beta) \leq d(\tilde{\alpha}, \tilde{\beta})$.

3.5. Proposition. Suppose $M$ is a space with $K \leq 0, \alpha(t)$ and $\beta(t)$ are geodesics. Then

$$
2 d[\alpha(0), \beta(0)]-4 \leq d(\alpha, \beta) \leq 2 d[\alpha(0), \beta(0)]+4 .
$$

Suppose $X$ is a Hadamard space. Recall the Busemann function $B=$ $B(\alpha, x)$, where $\alpha$ should be a geodesic and $x \in X$. (See Figure 7.)

$$
L_{t}=t+B+\varepsilon, \quad \lim _{t \rightarrow+\infty} \varepsilon=0 .
$$

The Busemann function is continuous with respect to $\alpha$ and $x$. See [Hu, 4.3].

3.6. Theorem. For a Hadamard space $X$, the following two conditions are equivalent.

(1) For any two asymptotic geodesics $\alpha$ and $\beta, d($ Image $\alpha$, Image $\beta)=0$.

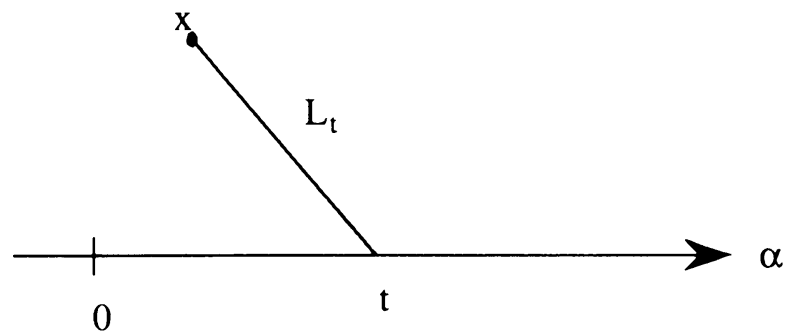

Figure 7 
(2) For any two asymptotic geodesics $\alpha$ and $\beta$, in $G(X)$,

$$
\lim _{\tau \rightarrow+\infty} d\{\tau \cdot \alpha,[\tau+B(\alpha, \beta(0))] \cdot \beta\}=0 .
$$

In fact the term on the left-hand side must monotonically decrease to zero.

3.7. Definition. Suppose $M$ is a space with $K \leq 0$ such that its universal covering satisfies the above conditions, then we say that the geodesic flow of $M, G(M)$, is weakly Anosov.

3.8. Theorem. Suppose $M$ is a compact space with $K \leq 0$ such that there is no totally geodesic $\mathbb{R} \times[a, b]$ immersed in it, then the geodesic flows of both $M$ and $M \times \mathbb{R}$ are weakly Anosov. Or suppose $M$ is a space with $K \leq \varepsilon<0$, then the geodesic flow of $M$ is weakly Anosov.

\section{THE SPACE OF RAYS AND NEAR HOMEOMORPHISMS}

Let $X$ be a Hadamard space. Define $R(X)=\{[0,+\infty) \rightarrow X$ isometry $\}$. We have an obvious 1-1 correspondence $R(X) \leftrightarrow X \times \partial X$. The topology of $R(X)$ is defined by this identification. Let $M$ be a space with $K \leq 0$. Define $R(M)=\{[0,+\infty) \rightarrow M$ local isometry $\}$. Suppose $X \rightarrow X / \Gamma=M$ is the universal covering of $M$. Then $R(X) \rightarrow R(X) / \Gamma=R(M)$ is the universal covering of $R(M)$, which defines the topology of $R(M)$. Then $R(M) \rightarrow M$ is a fiber bundle with fiber $\partial X$.

For a space $M$ with $K \leq 0, r \geq 0$, we also define $R_{r}(M)=\{[-r,+\infty) \rightarrow$ $M$ local isometry\}. It is left to the reader to verify that $G(M)=\lim R_{r}(M)$.

We will now investigate the possibility of approximating the canonical map $G(M) \rightarrow R(M)$ by homeomorphisms. First we review two theories of near homeomorphisms in topology.

Let $M^{n} \stackrel{f}{\rightarrow} N^{n}$ be a continuous proper surjective map between $n$-manifolds $N$ having a metric. $f$ is a near homeomorphism if for any continuous map $M \stackrel{\varepsilon}{\rightarrow}(0,+\infty)$, there is homeomorphism $M \stackrel{g}{\rightarrow} N$ such that $d(f x, g x) \leq$ $\varepsilon(x), x \in M . f$ is a cell-like map if, the inverse of any point of $N$ by $f$, is contractible in any neighborhood of itself in $M$.

4.1. Theorem (L. C. Siebenmann [Si]) $(n \geq 5) . f$ is a near homeomorphism if and only if it is cell-like.

A result of $\mathrm{M}$. Brown in [B] says that the inverse limit of near homeomorphisms of compact metric spaces is a near homeomorphism. We shall generalize this result for a noncompact case. A sketchy proof is included. First we define a measure to be any continuous map $X \stackrel{\varepsilon}{\rightarrow}(0,+\infty)$. A map $X \stackrel{f}{\rightarrow} Y$, $Y$ having a metric, is a near homeomorphism if for any measure $\varepsilon$ of $X$, there is homeomorphism $X \stackrel{g}{\rightarrow} Y$ such that $d(f x, g x) \leq \varepsilon(x), x \in X$. And $f$ is a homogeneous near homeomorphism if for any constant $\varepsilon>0$ there is homeomorphism $X \stackrel{g}{\rightarrow} Y$ such that $d(f x, g x) \leq \varepsilon, \forall x \in X$.

4.2. Theorem. Consider $X_{0} \stackrel{f_{1}}{\leftarrow} X_{1} \stackrel{f_{2}}{\leftarrow} X_{2} \leftarrow \cdots$, where $X_{i}$ are locally compact metric spaces and $f_{i}$ are continuous proper near homeomorphisms. Then $f_{1} f_{2} \cdots=f$ is a homogeneous near homeomorphism. 
Proof. (1) Suppose that $X \stackrel{f}{\rightarrow} Y$ is a continuous map between locally compact metric spaces and $\varepsilon$ is a measure for $Y$. Then there is a measure $\delta$ for $X$ such that

$$
d(x, y) \leq \delta(x) \Rightarrow d(f x, f y) \leq \varepsilon(f x) .
$$

(2) Take any constant $\varepsilon>0$. We can find homeomorphisms $g_{i}: X_{i} \rightarrow X_{i-1}$ and measures $a_{i}$ for $X_{i}$ such that

$$
d\left(g_{i_{0}} \cdots g_{i-1} f_{i}, g_{i_{0}} \cdots g_{i}\right) \leq a_{i} .
$$

We may assume that $a_{i} \leq \varepsilon / 2^{i-1}$. There are measures $b_{i}$ for $X_{i}$ such that

$$
x, y \in X_{i}, \quad d(x, y) \leq b_{i}(x) \Rightarrow d\left(f_{i_{0}} \cdots f_{i} x, f_{i_{0}} \cdots f_{i} y\right) \leq 1 / i .
$$

(3) We can further assume that

$$
\sum_{k=i+1}^{\infty} a_{k} f_{k+1} f_{k+2} \cdots \leq \frac{1}{2} b_{i} f_{i+1} f_{i+2} \cdots .
$$

Write $a_{i} f_{i+1} \cdots=\varepsilon_{i}$ and $b_{i} f_{i+1} \cdots=\delta_{i}$.

(4) In conclusion, we have homeomorphisms $g_{i}: X_{i} \rightarrow X_{i-1}$ and measures $\varepsilon_{i}$ for $X_{\infty}$ such that

$$
d\left(g_{i_{0}} \cdots g_{i-1} f_{i}, g_{i_{0}} \cdots g_{i}\right) f_{i+1} f_{i+2} \cdots \leq \varepsilon_{i}
$$

and $\sum_{i=1}^{\infty} \varepsilon_{i} \leq \varepsilon$.

We also have measures $\delta_{i}$ for $X_{\infty}$ such that

$$
d\left(f_{i+1} f_{i+2} \cdots x, f_{i+1} f_{i+2} \cdots y\right) \leq \delta_{i}(x) \Rightarrow x=y,
$$

and we have the relation

$$
\sum_{k=i+1}^{\infty} \varepsilon_{k} \leq \frac{1}{2} \delta_{i} .
$$

(5) Consider $g_{1} \cdots g_{i-1} f_{i} f_{i+1} \cdots$. It has limit which is denoted as $g_{1} g_{2} \ldots$. It is a homeomorphism and $d\left(f_{1} f_{2} \cdots, g_{1} g_{2} \cdots\right) \leq \varepsilon$.

4.3. Now go back and consider a space $M$ with $K \leq 0$. Assume the following additional conditions:

(1) For $x \in X$, the universal covering, $r>0, E_{x}(r) \cong E^{n}, n \geq 3$.

(2) For $x \in X, 0<r<R$, the canonical map $S_{x}(R) \rightarrow S_{x}(r)$ is a near homeomorphism.

Since $\partial X=\lim S_{x}(r)$ by Proposition 2.2.4, $\partial X \cong S^{n-1}$ by Brown's theorem. Then $R(M)$ is a fiber bundle over the manifold $M^{n}$ with fiber $S^{n-1}$. In particular $R(M)$ is a $(2 n-1)$-manifold, and the same holds for $R_{r}(M)$, $r \in \mathbb{R}$. Note that for $x \in X, 0<r<R, S_{x}(R) \rightarrow S_{x}(r)$ must be cell-like. We see that $R_{R}(M) \rightarrow R_{r}(M)$ is continuous, proper, surjective and cell-like. By Siebenmann's result, $R_{R}(M) \rightarrow R_{r}(M)$ is a near homeomorphism. But $G(M)=\lim ^{\longleftarrow} R_{r}(M)$. By 4.2, we get the following result.

4.4. Theorem. Suppose that $M$ is an n-manifold with $K \leq 0$ and suppose condition 4.3. Then $G(M) \rightarrow R(M)$ is a near homeomorphism.

\section{THE CONTROLLED $h$-COBORDISM THEOREM}

Suppose $W$ is a manifold with boundaries $M$ and $N$, and $M \hookrightarrow W, N \hookrightarrow$ $W$ are homotopy equivalences. Then there are $p_{t}, q_{t}: W \rightarrow W, 0 \leq t \leq 1$, 
strong deformations of $W$ to $M$ and to $N$. Set $p=p_{1}: W \rightarrow M$. Also suppose that everything is trivial outside a compact set of $M . W$ is called an $h$-cobordism. The following curves in $M$ are called associated curves of $W: p \circ p_{t}(x), 0 \leq t \leq 1, x \in W, p \circ q_{t}(x), 0 \leq t \leq 1, x \in W$.

Suppose $B \subset M, B \times[0,1] \stackrel{P}{\rightarrow} W$ is an embedding such that $\left.P\right|_{B \times 0}=$ Id, $P(B \times 1)=N, P(B \times(0,1)) \subset W^{0} . P$ is called a product structure for $W$ over $B$. The following are associated curves of $P: p P(x, t), 0 \leq t \leq 1$, $x \in B$.

Let $d$ be a metric of $M$. Then the maximum of the diameters of associated curves of $W$ is called the diameter of $W$. That of $P$ is the diameter of $P$.

5.1. Theorem (T. A. Chapman and S. Ferry [CF], F. Quinn [Q1]).

(1) Suppose $M^{n}$ is manifold, $n \geq 5, d$ is metric on $M, A, B \subset M$ are compact, $U$ is a neighborhood of $A$ in $M$. Then given $\varepsilon>0$ there is $\delta>0$ such that the following is true. For any h-cobordism $W$ over $M$, with product structure $P$ over $U$ and $\operatorname{diam}(W) \leq \delta$, $\operatorname{diam}(P) \leq \delta$, there is a product structure $Q$ for $W$ over $A \cup B$ such that $\left.Q\right|_{A}=P$, $\operatorname{diam}(Q) \leq \varepsilon$.

(2) Suppose $E \stackrel{\pi}{\rightarrow} M$ is a fiber bundle, $\operatorname{dim}(E) \geq 5$, the fiber $F$ is a compact manifold such that $\mathrm{Wh}\left(\pi_{1} F \times \mathbb{Z}^{k}\right)=0, k=0,1,2, \ldots$. Substitute $A, B, U$ by $\pi^{-1} A, \pi^{-1} B, \pi^{-1} U$, consider h-cobordisms over $E$, measure diameters in $M$. Then (1) is still true.

\section{STRATIFIED DECOMPOSITIONS FOR TOPOLOGICAL MANIFOLDS}

We will be using topological transversality. For the concept of microbundle, see [Mi]. For the concept of microbundle transversality, see [KS, p. 91]. Suppose $X$ is a metric space and $X \times[0,1] \stackrel{H_{f}}{\rightarrow} X$ is a continuous map, $H_{0}=\mathrm{Id}$, then define

$$
\operatorname{Supp}(H)=\overline{\left\{x \in X: H_{t} x \neq x\right\}}, \quad r(H)(x)=\max _{0 \leq t \leq 1} d\left(x, H_{t} x\right) .
$$

According to study of Kirby and Siebenmann in [KS, p. 91] and to recent study of Quinn in [Q2], we have the following result.

6.1. Theorem. Suppose that $M$ is a manifold, $X \subset M$ is a closed submanifold, $Y \subset M$ is a closed submanifold with normal microbundle $\nu, A, B \subset M$ are closed sets, and $X$ is transverse to $\nu$ near $A$. Then there is an ambient isotopy $H_{t}$ such that $H_{1}(X)$ is transverse to $\nu$ near $A \cup B . H_{t}$ is small and its support is in a small neighborhood of $B$ and outside a small neighborhood of $A$.

6.2. Theorem. Suppose that $M$ is a manifold, $X, Y, A$ are closed sets, $X-A$ and $Y-A$ are submanifolds, $Y-A$ has a normal microbundle $\nu$. Take any continuous map $M \stackrel{e}{\rightarrow}(0,+\infty)$ and any open neighborhood $U$ of $X$ in $M$. Then there is an ambient isotopy $H$ with the following properties:

(1) $\operatorname{Supp}(H) \subset U$,

(2) $r(H) \leq \varepsilon$,

(3) $\left.H\right|_{A} \equiv$ Id ,

(4) $H_{1}(X-A)$ is transverse to $\nu$.

Proof. Figure 8 indicates our idea which I heard of from L. E. Jones during a discussion. 


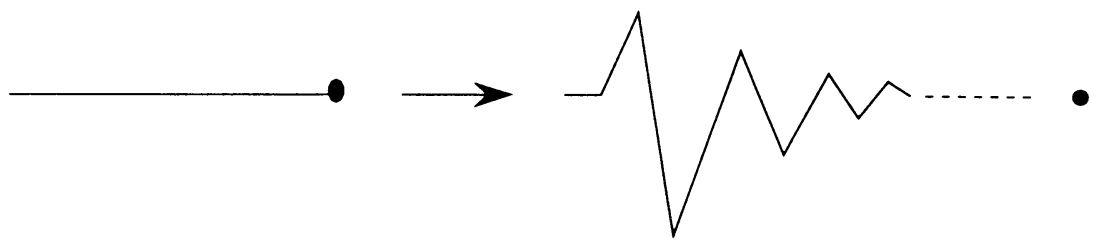

FIGURE 8

First separate $(0,+\infty)$ into $I_{1}=[1,+\infty), I_{i}=[1 / i, 1 / i-1]$. For each $I_{i}$, take an open interval $J_{i}, I_{i} \subset J_{i} \subset(0,+\infty)$ such that $J_{i} \cap J_{j} \neq \varnothing \Leftrightarrow|i-j| \leq 1$.

Define $f(x)=d(x, A): M \rightarrow \mathbb{R}$. Note that $f(x)$ is continuous, $f(x)=$ $0 \Leftrightarrow x \in A$. Write $f^{-1}\left(I_{i}\right) \cap X=A_{i}, f^{-1}\left(J_{i}\right) \cap U=U_{i}$.

Take any $i \in\{1,3,5, \ldots\}$, apply the above-mentioned theorem of KirbySiebenmann-Quinn in $M-A$, we have

$$
\left\{\begin{array}{l}
H^{i}: \text { Ambient isotopy in } M-A \\
\operatorname{Supp}\left(H^{i}\right) \subset U_{i} \\
r\left(H^{i}\right) \leq \min (1 / i, \varepsilon / 2) \\
H_{1}^{i}(X-A) \text { near } A_{i} \text { is transverse to } \nu .
\end{array}\right.
$$

Now $\operatorname{Supp}\left(H^{i}\right) \subset M-A$ closed, $\operatorname{Supp}\left(H^{i}\right) \subset U_{i} \subset f^{-1}\left(J_{i}\right) \subset f^{-1}\left(\bar{J}_{i}\right)$, $\overline{\operatorname{Supp}\left(H^{i}\right)} \subset f^{-1}\left(\bar{J}_{i}\right), o \notin \bar{J}_{i}, f^{-1}\left(\bar{J}_{i}\right) \subset M-A, \overline{\operatorname{Supp}\left(H^{i}\right)} \subset M-A$, $\overline{\operatorname{Supp}\left(H^{i}\right)}=\operatorname{Supp}\left(H^{i}\right), \operatorname{Supp}\left(H^{i}\right) \subset M$ is closed. So $H^{i}$ is trivially extended to $M$. We still denote the extension as $H^{i}$.

Consider $\widetilde{H}=H^{1} H^{3} H^{5} \ldots$. We want to show that $\widetilde{H}$ is an ambient isotopy. In fact the following two arguments are enough to do so. Argument 1: Suppose $\left(t_{i}\right) \subset[0,1], t_{i} \rightarrow t,\left(x_{i}\right) \subset M, x_{i} \rightarrow x \in A$. Then for any finite union $\bigcup f^{-1}\left(I_{i}\right), x_{i}$ will leave it eventually. We see that $d\left(x_{i}, \widetilde{H}_{t_{i}}\left(x_{i}\right)\right) \rightarrow 0$. So $\widetilde{H}_{t_{i}}\left(x_{i}\right) \rightarrow x$. Argument 2: Fix $t \in[0,1]$. Suppose $\left(x_{i}\right) \subset M, x \in A$, $\widetilde{H}_{t}\left(x_{i}\right) \rightarrow x$. Suppose there is $B=f^{-1}\left(\bar{J}_{i_{1}}\right) \cup \cdots \cup f^{-1}\left(\bar{J}_{i_{k}}\right)$ from which $\left(x_{i}\right)$ does not eventually leave. Since $\left.H_{t}\right|_{B}$ is a homeomorphism of $B$, we see that $x \in B$. This is impossible. So $\left(x_{i}\right)$ does leave any finite union $\cup f^{-1}\left(J_{i}\right)$ eventually. So $d\left(x_{i}, \widetilde{H}_{t}\left(x_{i}\right)\right) \rightarrow 0$ and $x_{i} \rightarrow x$.

For any $i \in\{2,4,6, \ldots\}$, again apply 6.1 in $M-A$. We have

$$
\left\{\begin{array}{l}
H^{i}: \text { Ambient isotopy in } M-A, \\
\operatorname{Supp}\left(H^{i}\right) \subset \widetilde{H}_{1}\left(U_{i}\right) \text {-neighborhood of } \widetilde{H}_{1}\left(A_{i-1}\right) \text {-neighborhood of } \widetilde{H}_{1}\left(A_{i+1}\right), \\
r\left(H^{i}\right) \leq \min (1 / i, \varepsilon / 2), \\
H_{1}^{i} \widetilde{H}_{1}(X-A) \text { is transverse to } \nu \text { near } \widetilde{H}_{1}\left(A_{i}\right) .
\end{array}\right.
$$

Again $\operatorname{Supp}\left(H^{i}\right) \subset M$ is closed and $H^{i}$ is trivially extended to $M . \widehat{H}=$ $H^{2} H^{4} H^{6} \ldots$. It is an isotopy of $M . H=\widetilde{H} * \widehat{H}$. This is what we want.

6.3. Definition. Let $M$ be a manifold. Suppose that $M \supset X \supset X_{1} \supset \cdots \supset$ $X_{k} \supset \phi$ is a finite descending series of closed sets, $X_{i}-X_{i+1}, 0 \leq i \leq k$, are submanifolds of $M$, and $\operatorname{dim}\left(X_{i}-X_{i+1}\right)$ is nonincreasing, then call $X$ a stratified set. Denote $\operatorname{dim}(X)=\operatorname{dim}\left(X-X_{1}\right)$. If all $X_{i}-X_{i+1}$ have normal microbundles, then say that $X$ has a normal microbundle. 
6.4. Definition. Let $M^{n}$ be a manifold, $\{X\}$ is a collection of stratified sets. They are said to be in general position if for any $\left\{X_{1}, \ldots, X_{k}\right\} \subset\{X\}, \cap_{i=1}^{k} X_{i}$ is inside a stratified subset $Y$ of some $X_{i_{0}}$, with

$$
\operatorname{dim} Y \leq \operatorname{dim}\left(X_{1}\right)+\cdots+\operatorname{dim}\left(X_{k}\right)-(k-1) n .
$$

6.5. Lemma. Let $M$ be a manifold, $X_{1}, \ldots, X_{k}$ be stratified sets with normal microbundles, and $X$ a closed submanifold. Then there is an ambient isotopy, small and concentrated near $X$, and a stratification of $X$ such that $X$ becomes in general position with any stratified subset of any $X_{i}$. If $X$ has a normal microbundle then the stratification also has a normal microbundle.

Proof. Collect in ascending order all dimension zero stratified subsets of $X_{1}$, then $X_{2}, \ldots$ and lastly $X_{k}$. Then do the same thing in dimension one, and so on. Denote the final collection as $Y_{1}, Y_{2}, \ldots, Y_{l}$.

Take $Y_{1}$. If $X \cap Y_{1}=\varnothing$, then go on to $Y_{2}$. Suppose $X \cap Y_{1} \neq \varnothing$. Apply 6.2 and we may assume that $X \cap Y_{1}=Z_{1}, Z_{1} \subset X$ is a submanifold with normal microbundle, $\operatorname{dim}\left(Z_{1}\right)=\operatorname{dim}(X)+\operatorname{dim}\left(Y_{1}\right)-n$. Suppose $Y_{1} \cdots Y_{i}$ are handled and we obtain stratification $X \supset Z_{j} \supset \cdots \supset Z_{1} \supset \varnothing$, where $Z_{k}-Z_{k-1}$ are submanifolds with normal microbundles in $X, X \cap\left(Y_{1} \cup \cdots \cup Y_{i}\right)=Z_{j}$, $\operatorname{dim}\left(Z_{j}\right)=\operatorname{dim}(X)+\operatorname{dim}\left(Y_{i_{0}}\right)-n$. Consider $Y_{i+1}$. If $X \cap Y_{i+1} \subset Y_{1} \cup \cdots \cup Y_{i}$, then go on to $Y_{i+2}$. Suppose $X \cap Y_{i+1} \not \subset Y_{1} \cup \cdots \cup Y_{i}$. Note that $X-Y_{1} \cup \cdots \cup Y_{i}=$ $X-Z_{j}, Y_{i+1}-Y_{1} \cup \cdots \cup Y_{i}$ is a submanifold with normal microbundle in $M$. Apply 6.2 and we see that we may assume that

$$
\left(X-Y_{1} \cup \cdots \cup Y_{i}\right) \cap\left(Y_{i+1}-Y_{1} \cup \cdots \cup Y_{i}\right)=A \text {, }
$$

$A$ is a submanifold with normal microbundle in $X, \operatorname{dim}(A)=\operatorname{dim}(X)+$ $\operatorname{dim}\left(Y_{i+1}\right)-n$. Note that $A \cap Z_{j}=\varnothing, X \cap\left(Y_{1} \cup \cdots \cup Y_{i} \cup Y_{i+1}\right)=Z_{j} \cup A$. Define $A \cup Z_{j}=Z_{j+1}$. Finally $Y_{l}$ can be handled.

6.6. Theorem. Let $M$ be a manifold, each $\Sigma_{i}$ be a finite collection of stratified sets with normal microbundles in general position, $1 \leq i \leq k$, and $X$ a closed submanifold. Then there is an ambient isotopy, small and concentrated near $X$, and a stratification of $X$ such that $\Sigma_{i} \cup\{X\}$ is in general position, $1 \leq i \leq k$. If $X$ has normal microbundle, then the stratification also has one.

Proof. Consider $\Sigma_{i} \cup \cdots \cup \Sigma_{k}$ and $X$. Apply 6.5.

6.7. Let $M^{n}$ be manifold, $\left\{E^{n}\right\}$ a locally finite collection of cells. Assume that for any $\left\{S_{1}, \ldots, S_{n+1}\right\} \subset\left\{S_{i}\right\}=\left\{\partial E^{n-1}\right\}, S_{1} \cap \cdots \cap S_{n+1}=\varnothing$ : For example, we can apply 6.6 to put $\left\{S_{i}\right\}$ in general position. Consider $\left\{M-E_{i}^{n}\right\} \cup\left\{\operatorname{Int}\left(E_{i}^{n}\right)\right\}$ and take some sets from it to obtain an intersection. Let $\{U\}$ be the collection of smallest but nonempty intersections. We have $M-\bigcup S_{i}=\biguplus U$, where $\biguplus$ is the notation for disjoint union. Each $e=U$ is defined to be an $n$-cell. Note that $\partial e \subset \bigcup S_{i}$. Take any $\left\{S_{1}, \ldots, S_{k}\right\} \subset\left\{S_{i}\right\}$. Write $M-\bigcup_{i \neq 1, \ldots, k} S_{i}$ into, in the same way, $M-\bigcup_{i \neq 1, \ldots, k} S_{i}=\biguplus U$. Each $e=S_{1} \cap \cdots \cap S_{k} \cap U$ is defined to be a cell with $\operatorname{dim}(e)=n-k$. Denote $\bigcup \cap^{1}=\bigcup S_{i}, \cup \bigcap^{2}=\bigcup_{i \neq j} S_{i} \cap S_{j}$, and so on.

$$
\begin{gathered}
S_{1} \cap \cdots \cap S_{k}-\bigcup_{i \neq l \cdots k} S_{i}=\biguplus S_{1} \cap \cdots \cap S_{k} \cap U, \\
S_{1} \cap \cdots \cap S_{k}-\bigcup \bigcap^{k+1}=\biguplus S_{1} \cap \cdots \cap S_{k} \cap U .
\end{gathered}
$$


Note that for any $e=S_{1} \cap \cdots \cap S_{k} \cap U$, $\partial e \subset \cup \cap^{k+1}$. And we have $\cup \bigcap^{k}-\cup \bigcap^{k+1}=\biguplus e$, all $(n-k)$-cells. We have obtained, in the following sense, a stratified decomposition for $M$.

6.8. Stratified decompositions. Definition: $M^{n}=\biguplus e$. Each $e$ is assigned an integer as dimension, $0 \leq \operatorname{dim}(e) \leq n$.

$$
\partial e \subset \bigcup_{\operatorname{dim}(\tilde{e}) \leq \operatorname{dim}(e)-1} \tilde{e} .
$$

And $\{\bar{e}\}$ is locally finite.

\section{A $C^{0}$ FOLIATED CONTROL THEOREM}

In this section we shall establish a long thin stratified decomposition and prove a topological foliated control result, that is the topological version of a smooth foliated control result of Farrell and Jones in [FJ1, $\S \S 6-8]$. This result can handle the geodesic flow $G$ of any PL manifold with $K \leq 0$. As we see in $\S 1$, there is geodesic singularity in this case. Because of that $G$ is not local. So it is hopeless for $G$ to have PL or $C^{+\infty}$ structure, it is just a topological foliation.

7.1. Suppose $M$ is an $n$-dimensional manifold with metric and with a unit speed 1-dimensional foliation structure and $X \subset M$ is a compact set such that any leaf intersecting $X$ has length $>l$.

Split neighborhood: Suppose $E^{n-1}$ is a transversal section, $\forall x \in E^{n-1}$ there is a compact interval $I_{x}$ in a leaf and suppose that $I_{x}$ is continuous. Then, for convenience, denote $I \times E^{n-1}=\bigcup_{x \in E^{n-1}} I_{x}$.

For any interval $I=[a, b]=(a+b) / 2+[-(b-a) / 2,(b-a) / 2], r>$ 0 , denote $I(r)=(a+b) / 2+[-(b-a) / 2 \cdot r,(b-a) / 2 \cdot r]$. Take a finite collection of split neighborhood $\left\{I \times E^{n-1}\right\}$ such that length $(I)=l$ for all $I$ and $\left\{I(1 / 40 n) \times E^{n-1}(1 / 2)\right\}$ covers $X$. Also assume that for any two in $\left\{I(1 / 2) \times E^{n-1}\right\}$, the intersection is some split set $P \times Q=\bigcup_{x \in Q} P_{x}$.

We want to reconstruct $\left\{I(1 / 40 n) \times E^{n-1}\right\}$ by induction. The following are by induction hypotheses:

(1) $\{A \times \widetilde{B}\}$ are finitely many split neighborhoods constructed out of

$$
\left\{I_{1}(1 / 40 n) \times E_{1}^{n-1}, \ldots, I_{i}(1 / 40 n) \times E_{i}^{n-1}\right\} \subset\left\{I(1 / 40 n) \times E^{n-1}\right\} .
$$

(2) length $(A) \leq l / 4$ for all $A$.

(3) Take any leaf of $M$ and consider the intersections of $\{A \times \widetilde{B}\}$ with the leaf. Any two different end points have distance $\geq l / 40 n$.

(4) Each $\widetilde{B}$ of $\{A \times \widetilde{B}\}$ can be shrunk to $B$ such that $\{A \times B\}$ covers $I_{1}(1 / 40 n) \times E_{1}^{n-1}(1 / 2) \cup \cdots \cup I_{i}(1 / 40 n) \times E_{i}^{n-1}(1 / 2)$.

Take $I(1 / 40 n) \times E^{n-1} \in\left\{I_{i+1}(1 / 40 n) \times E_{i+1}^{n-1}, \ldots\right\}$. Put a triangulation on $E^{n-1}$. Consider any simplex $\Delta$ and any $A \times \widetilde{B} \in\{A \times \widetilde{B}\}, I(1 / 2) \times E^{n-1} \cap$ $A \times \widetilde{B} \neq \varnothing . \Delta$ is canonically mapped to $\widetilde{B}$. Take a cell $\widehat{B}$ between $B$ and $\widetilde{B}$. (See Figure 9.)

We can require the triangulation to be so fine that if $\operatorname{Image}(\Delta) \cap \widehat{B} \neq \varnothing$, Image $(\Delta) \subset \widetilde{B}^{0}$. Thicken up $\Delta$ to $F^{n-1}$ such that if

$$
\text { Image }(\Delta) \subset \widetilde{B}^{0}, \quad \operatorname{Image}\left(F^{n-1}\right) \subset \widetilde{B}^{0}
$$

and if $\operatorname{Image}(\Delta) \cap \widetilde{B}=\varnothing, \operatorname{Image}\left(F^{n-1}\right) \cap \widehat{B}=\varnothing$. 


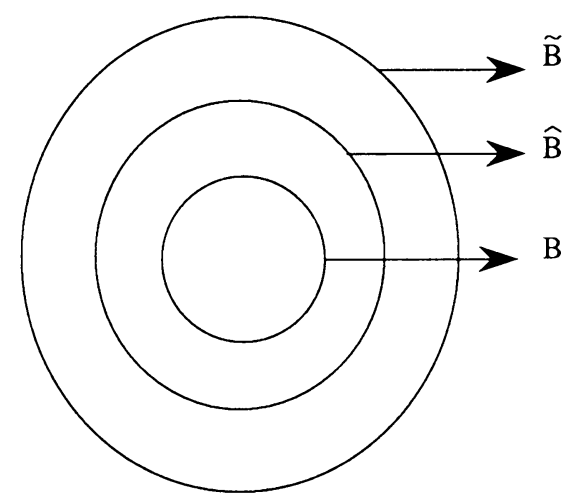

FIGURE 9
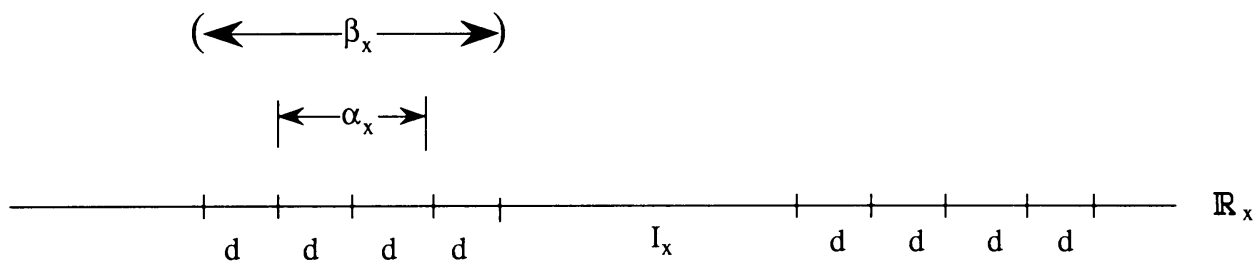

FIGURE 10

7.2. Lemma. Consider $\mathbb{R} \times \mathbb{R}^{n-1}, D^{n-1}$ represents an open disk. $\left\{I_{i} \times D_{i}^{n-1}\right\}$ is a finite collection of split neighborhoods such that in any leaf $\mathbb{R}_{x}$ any two different end points of $\left\{I_{i x}\right\}$ have distance $\geq d$. I $\times E^{n-1}$ is a split neighborhood, $r=$ length $(I) \geq d$. $E^{n-1}$ is canonically mapped into $D_{i}^{n-1}, \forall i$. Then when $E^{n-1}$ is small enough, there is a split neighborhood $J \times E^{n-1}$ such that $I \times E^{n-1} \subset$ $J \times E^{n-1} \subset I(r+8 d / r) \times E^{n-1}$ and that $\left\{I_{i} \times D_{i}^{n-1}\right\} \cup J \times E^{n-1}$ still has the property of $\left\{I_{i} \times D_{i}^{n-1}\right\}$.

Proof. (See Figure 10.) Note that when $E^{n-1}$ is small enough the following is true. Either there is no end point of $\left\{I_{i x}\right\}$ in the closed interval $\alpha_{x}, \forall x \in E^{n-1}$ : Then take the midpoint of $\alpha_{x}, x \in E^{n-1}$. Or an end point of $I_{i_{0} x}$ is in the open interval $\beta_{x}, \forall x \in E^{n-1}$ : Then take it. Do the same thing at the other side of $I$. For each $x \in E^{n-1}$, the two points chosen form an interval $J_{x} \cdot J \times E^{n-1}=\bigcup_{x \in E^{n-1}} J_{x}$.

Take any $k$-simplex $\Delta$ with thickening $F^{n-1}$. Consider $I(10 k+2 / 40 n)$ $\times F^{n-1}$. If Image $(\Delta) \cap \widehat{B}=\varnothing, \forall A \times \widetilde{B} \in\{A \times \widetilde{B}\}$, then pick $J \times F^{n-1}=$ $I(10 k+2 / 40 n) \times F^{n-1}$. Suppose $\{C \times \widetilde{D}\}$ is the collection of those $A \times \widetilde{B} \in$ $\{A \times \widetilde{B}\}$ such that $\operatorname{Image}(\Delta) \cap \widehat{B}=\varnothing$. Apply 7.2 with $\left\{C \times \widetilde{D}^{0}\right\}, d=l / 40 n$, and with $I(10 k+2 / 40 n) \times F^{n-1}$. We get a split neighborhood $J \times F^{n-1}$ such that

$$
I(10 k+2 / 40 n) \times F^{n-1} \subset J \times F^{n-1} \subset I(10(k+1) / 40 n) \times F^{n-1}
$$

and that $\left\{C \times \widetilde{D}^{0}\right\} \cup J \times F^{n-1}$ has the property (4) of the induction hypotheses. Treat all simplices. 
It is left for the reader to check that $\{A \times \widehat{B}\} \cup\left\{J \times F^{n-1}\right\}$ satisfies the induction hypotheses.

\subsection{Conclusion.}

(1) $\{A \times \widetilde{B}\}$ are finitely many split neighborhoods.

(2) length $(A) \leq l / 4$, for all $A$.

(3) Take any leaf of $M$; consider the intersection of $\{A \times \widetilde{B}\}$ with the leaf. Any two different end points have distance $\geq l / 40 \mathrm{n}$.

(4) Each $\widetilde{B}$ of $\{A \times \widetilde{B}\}$ can be shrunk to $B$ such that $\{A \times B\}$ covers $X$.

For each $A \times \widetilde{B} \in\{A \times \widetilde{B}\}$, construct a split neighborhood $[-l / 4, l / 4] \times \widetilde{B}$ such that $A \times \widetilde{B}$ and $[-l / 4, l / 4] \times \widetilde{B}$ have the same interval centers. We shall assume that the intersection of any two of $\{[-l / 4, l / 4] \times \widetilde{B}\}$ is some split set $P \times Q=U_{x \in Q} P_{x}$. For each $[-l / 4, l / 4] \times B$, cover $B$ by very small cells: We see that the following can be assumed. For any $[-l / 4, l / 4] \times \widetilde{B}$, $[-l / 4, l / 4] \times \widetilde{D} \in\{[-l / 4, l / 4] \times \widetilde{B}\},[-l / 4, l / 4] \times \widetilde{D} \cap[-l / 4, l / 4] \times \widetilde{B} \neq \varnothing$, consider the canonical map between $\widetilde{D}$ and $\widetilde{B}$, Image $(D) \cap B \neq \varnothing$, then Image $(D) \subset \widetilde{B}^{0}$. It is left for the reader to apply Theorem 6.6 to establish the following additional condition.

(5) Take any $[-l / 4, l / 4] \times B \in\{[-l / 4, l / 4] \times B\} .\{[-l / 4, l / 4] \times D\}$ is the collection from $\{[-l / 4, l / 4] \times B\}$ such that $\operatorname{Image}(D) \cap B \neq \varnothing$ in the abovementioned sense. Then in $\widetilde{B}^{0}, \partial B+\{\operatorname{Image}(D)\}$ is in general position, for which we mean Definition 6.4.

\subsection{We now arrange $\bigcup A \times B$ into a long thin stratified decomposition.}

Transversal cells. $\forall x \in \bigcup \partial A \times B$. Denote the union of those in $\{A \times B\}$ that contain $x$ as $U_{x}$. Denote by $\{C \times D\}$ the collection of those $A \times B \in\{A \times B\}$ such that $A \times B \cap U_{x} \neq \varnothing$. Assume that $x \in B \in\{\partial A \times B\}=\left\{\partial A^{+} \times B, \partial A^{-} \times\right.$ $B\}$. Project $\{D\}$ into $\widetilde{B}^{0}$. Denote $\{\operatorname{Image}(D)\}=\left\{E_{i}^{n-1}\right\} .\left\{S_{i}\right\}=\left\{\partial E_{i}^{n-1}\right\}$ is in general position in $\widetilde{B}^{0}$. These are by $7.3(5)$.

Consider the stratified cell $e$ that contains $x$. Recall how this cell is constructed following 6.7: $x \in S_{1} \cap \cdots \cap S_{k}-\bigcup_{i \neq 1, \ldots, k} S_{i} \cdot \widetilde{B}^{0}-\bigcup_{i \neq 1, \ldots, k} S_{i}=\biguplus U$, see 6.7. Take $U, x \in U$. Then $e=S_{1} \cap \cdots \cap S_{k} \cap U, \operatorname{dim}(e)=n-k-1$. Fix any $y \in e$. Suppose $C \times D \in\{A \times B\}, x \in C \times D$. Then Image $(D) \in\left\{E_{i}^{n-1}\right\}$, $x \in \operatorname{Image}(D)$. So $e \subset \operatorname{Image}(D)$. We see that $y \in C \times D$. On the other hand assume that $C \times D \in\{A \times B\}, y \in C \times D$. Then $C \times D \cap U_{x} \neq \varnothing$. So Image $(D) \in\left\{E_{i}^{n-1}\right\}, y \in \operatorname{Image}(D)$. So $e \in \operatorname{Image}(D)$. Then $x \in C \times D$. We see that $U_{x}=U_{y}$. So we produce for $y$ the same $\left\{E_{i}^{n-1}\right\}$, and hence the same $e$. We have $\bigcup \partial A \times B=\biguplus e$.

Consider any $y \in \partial e$. Take any $C \times D \in\{A \times B\}, x \in C \times D$. Then Image $(D) \in\left\{E_{i}^{n-1}\right\}, x \in \operatorname{Image}(D)$. Then $e \subset \operatorname{Image}(D)$ and $\bar{e} \subset \operatorname{Image}(D)$. We see that $y \in C \times D$, so $U_{x} \subset U_{y}$. We conclude that more than, or at least the same, $\left\{E_{i}^{n-1}\right\}$ are produced for $y$. On the other hand, $x \in S_{1} \cap \cdots \cap S_{k}-$

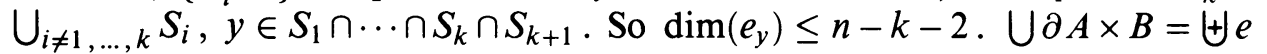
is a stratified decomposition in the sense of 6.8 .

Flow cells. $\forall x \in \bigcup A \times B-\bigcup \partial A \times B$. Take a nearest end point $z$ from $x$ of $\{A \times B\}$ in the leaf of $x$. Denote the union of those in $\{A \times B\}$ that contain $x$ as $U_{x} .\{C \times D\}$ is the collection of those $A \times B \in\{A \times B\}$ such 
that $A \times B \cap U_{x} \neq \varnothing$. Assume that $z \in B \in\{\partial A \times B\}$. Project $\{D\}$ into $\widetilde{B}^{0}$. Denote $\{\operatorname{Image}(D)\}=\left\{E_{i}^{n-1}\right\} \cdot\left\{S_{i}\right\}=\left\{\partial E_{i}^{n-1}\right\}, z \in S_{1} \cap \cdots \cap S_{k}-\bigcup_{i \neq 1, \ldots, k} S_{i}$. $\widetilde{B^{0}}-\bigcup_{i \neq 1, \ldots, k} S_{i}=\biguplus U$. Pick out $U, z \in U$. Denote $b=S_{1} \cap \cdots S_{k} \cap U$. Denote the intersection of those in $\{A \times B\}$ that contain $x$ as $P \times Q$. For convenience, identify $Q$ in $\widetilde{B}^{0}$. Then $Q$ is the intersection of several $E_{i}^{n-1} \in$ $\left\{E_{i}^{n-1}\right\}$ that contain $z$. So $b \subset Q, \forall \omega \in b$, define $a_{\omega}=P_{\omega}^{0} . a \times b$ is defined to be a flow cell. Designate $\operatorname{dim}(a \times b)=n-k$.

The following are true: $\bigcup A \times B-\bigcup \partial A \times B=\biguplus a \times b . \bar{b}$ is covered by transversal cells with dimensions $\leq n-k-1 . \bar{a} \times \partial b \cap \bigcup \partial A \times B$ is covered by transversal cells with dimensions $\leq n-k-2$, and $\bar{a} \times \partial b \cap(\bigcup A \times B-\bigcup \partial A \times B)$ is covered by flow cells with dimensions $\leq n-k-1$.

Long thin stratified decomposition. So $\bigcup A \times B=\biguplus e \biguplus a \times b$ is a stratified decomposition in the sense of 6.8 .

7.5. We now collect lemmas to be used for the foliated control result. Suppose $M$ is a manifold with a 1-dimensional foliation structure and a metric. $\alpha(t)$, $0 \leq t \leq 1$, is a curve in $M, l$ and $a$ are positive numbers, and $I$ is a leaf segment of the foliation such that length $(I) \leq l$ and $d[\alpha(t), I] \leq a, 0 \leq t \leq L$. Then we say that the curve has diameter $\leq(l, a)$. If there is a collection of curves such that every curve has diameter $\leq(l, a)$, then we say that the collection has diameter $\leq(l, a)$. If $W$ is an $h$-cobordism over $M$ and $P$ is a product for $W$, then their diameters are those of their respective assoliated curves.

7.5.1. Lemma. Let $d$ and $D$ be unit speed metrics on foliated $M . X \subset M$ compact, $l>0$. Then for any $\varepsilon>0$, there is $\delta>0$ such that for any collection of curves $\{\alpha\}$ in $X$ which is $(l, \delta)$-controlled, i.e., diameter $\leq(l, \delta)$ in terms of $d,\{\alpha\}$ is $(l, \varepsilon)$-controlled in terms of $D$.

Proof. Suppose this is not the case. Then we have intervals in leaves $I_{i}$ and $x_{i}, d\left(x_{i}, I_{i}\right) \leq 1 / i$, but $D\left(x_{i}, I_{i}\right) \geq \varepsilon, i=1,2,3, \ldots . I_{i}$ and $x_{i}$ are in a compact set. We may well suppose that $x_{i} \rightarrow x, I_{i} \rightarrow J$. Then $d(x, J)=$ $0: x \in J$. But $D(x, J) \geq \varepsilon: x \notin J$. This is impossible.

7.5.2. Lemma. Consider $\mathbb{R} \times M \stackrel{l \text { Id } \times \text { Id }}{\longrightarrow} \mathbb{R} \times M, l>0, M$ a metric space. Then for any collection of curves $\{\alpha\}$ in $\mathbb{R} \times M$ which is $(\varepsilon, a)$-controlled, $(l \mathrm{Id} \times \mathrm{Id})\{\alpha\}$ is $(\varepsilon l, \max (1, l) \cdot a)$-controlled .

Proof. Recall that for $(u, x),(v, y) \in \mathbb{R} \times M$,

$$
\sqrt{l^{2}|u-v|^{2}+d(x, y)^{2}} \leq \max (1, l) \cdot \sqrt{|u-v|^{2}+d(x, y)^{2}} .
$$

7.5.3. Lemma. $I=[-1,1], n \geq 5$. Let $M^{n-1}$ be manifold, $A, B \subset M$ compact, $A_{0}$ compact with neighborhood $A$. Then for any $\varepsilon, a>0$, there are $\delta, b>0$, where $\delta$ depends only on $n$ and $\varepsilon$, such that the following is true. For any $h$-corbordism $W$ over $\mathbb{R} \times M$ with product structure $P$ over $I \times A$ such that $d(W) \leq(\delta, b), d(P) \leq(\delta, b)$, there is a product structure $Q$ over $I \times A_{0} \cup I(1 / 2) \times B$ such that $\left.Q\right|_{I \times A_{0}}=P, d(Q) \leq(\varepsilon, a)$.

Proof. Cover $A_{0} \cup B$ by a compact simplicial complex $K$. Make sure that any simplex that intersects $A_{0}$ is inside $A^{0}=\operatorname{Int}(A)$. And for any simplex $\Delta$ with 
thickening $T$, if $\Delta \subset A^{0}, T \subset A^{0}$, if $\Delta \cap A^{0 \prime} \neq \varnothing, T \cap A_{0}=\varnothing$. We now construct $Q$. Suppose $0,1, \ldots, i-1$ simplices are treated. For an $i$-simplex $\Delta$ with thickening $T$, if $\Delta \subset A^{0}$, take $\left.P\right|_{I \times T}$. Suppose $\Delta \cap A^{0 \prime} \neq \varnothing$. Consider the statement 7.5.3, substitute $B$ by $T, I$ by $I(1-(i-1) / 2(n-1))$, and $I(1 / 2)$ by $I(1-i / 2(n-1))$. This is equivalent to the following special case.

Denote $I=[-1,1], I^{k}=X_{k} I, I^{k}(r)=X_{k} I(r)$. Special case of the lemma:

$$
\begin{gathered}
M=\mathbb{R}^{n-1}, \quad A=\left(I^{i}-I^{i}(1 / 2)\right) \times I^{n-i-1}, \\
A_{0}=\left(I^{i}-I^{i}(3 / 4)\right) \times I^{n-i-1}(1 / 2), \quad B=I^{i}(3 / 4) \times I^{n-i-1}(1 / 2),
\end{gathered}
$$

but the metric on $\mathbb{R}^{n-1}$ is any metric. Apply 7.5.1 and we may assume that the metric on $\mathbb{R}^{n-1}$ is standard. Then [FJ1, Proposition 6.6] implies the lemma in this case.

7.5.4. Lemma. Lemma 7.5.3 is still true when we substitute $I \times A$ for $I \times A \cup$ $(I-I(1 / 2)) \times M, I \times A_{0}$ for $I \times A_{0} \cup(I-I(3 / 4)) \times M$ and $I(1 / 2) \times B$ for $I(3 / 4) \times B$.

The proof similar to the above one is left for the reader. We now establish the foliated control result.

7.6. Theorem $(n \geq 5) . M$ is an $n$-manifold with metric and with a unit speed 1-dimensional foliation structure, $X \subset M$ is a compact set such that any leaf intersecting $X$ has length $>l$. Then for any $\varepsilon, a>0$, there are $\delta, b>0$, where $\delta$ depends only on $n$ and $\varepsilon^{\prime}$ such that the following is true. For any $h$-cobordism $W$ over $M$ with $d(W) \leq(\delta l, b)$, there is product structure $P$ over $X$ with $d(P) \leq(\varepsilon l, a)$.

Proof. We shall continue to use notations $\{A \times \widetilde{B}\}$ of 7.3 and $\bigcup A \times B=$ $\biguplus e \biguplus a \times b$ of 7.4 .

Denote $I=[-1,1]$. Denote $\{\partial A \times \widetilde{B}\}=\{\widetilde{B}\}$. First consider $\cup I(l / 100 n) \times$ $\widetilde{B}^{0}$ which is a subfoliation in $M$. Thicken up $\bigcup \partial A \times B=\biguplus e$ in $\bigcup \partial A \times \widetilde{B}^{0}$. (For each $e$ there are $K_{e}$ and $T_{e}$ compact, $K_{e} \subset T_{e}$ is a neighborhood, $\cup e \subset$ $\left.\bigcup K_{e}, \operatorname{dim}(e)=\operatorname{dim}(\tilde{e}): T_{e} \cap T_{\tilde{e}}=\varnothing.\right)$ Suppose $0,1, \ldots, i-1$ transversal cells are treated and we have product structure over

$$
\bigcup_{d(e) \leq i-1} I\left(\frac{l}{100 n}\left(1-\frac{i-1}{2(n-1)}\right)\right) \times T_{e} .
$$

Take any transversal cell $e, \operatorname{dim}(e)=i$. Assume that $e \subset B \subset \widetilde{B}^{0}$. Then we have thickening $T$ of $e$ in $\widetilde{B}^{0}$. Apply 7.5.1 to change the metric on $I(l / 100 n) \times$ $\widetilde{B}^{0}$ to a product metric. Apply 7.5 .2 to shrink $I(l / 100 n) \times \widetilde{B}^{0}$ by $1 / 100 n$ in the flow direction to $I \times \widetilde{B}^{0}$. Then apply 7.5.3, in whose statement substitute $I$ by $I(i / 2(n-1))$ and $I(1 / 2)$ by $I((i-1) / 2(n-1))$, to extend the product structure over $I(i / 2(n-1)) \times T$. Apply 7.5 .2 to expand $I \times \widetilde{B}^{0}$ back to $I(l / 100 n) \times \widetilde{B}^{0}$. And finally apply 7.5.1 to change the metric back to that on $M$. We have extended the product structure over

$$
I\left(\frac{l}{100 n}\left(1-\frac{i}{2(n-1)}\right)\right) \times T .
$$

In the end we get a product structure over $\bigcup I(l / 200 n) \times T$. 
Consider flow cells. Suppose that $R$ is a neighborhood of

$$
\bigcup I\left(l / 200 n \cdot 1 / 8^{i-1}\right) \times B \bigcup_{\operatorname{dim} \leq i-1} a \times b
$$

and we have constructed product structure over $R$. Take any $a \times b, \operatorname{dim}(a \times b)$ $=i$. Since $\bar{b}$ can be small, we can have $I(r) \times U, \bar{b} \subset U \cong \mathbb{R}^{n-1}, l / 100 n \leq$ $r \leq l / 8$,

$$
\left(I(r)-I\left(r-l / 200 n \cdot 1 / 8^{i-1} \cdot 1 / 2\right)\right) \times U \subset R .
$$

$a \times b-R^{0}=\overline{a \times b}-R^{0}$ is compact. Denote its projection to $b$ as $K \subset b$. Take a compact neighborhood $N$ of $K$ in $U$. Since $K$ is compact, $K \subset b$ and $N$ can be very close to $K$, the following can be assumed: $I(r) \times N$ is disjoint from flow cells with $\operatorname{dim} \leq i-1$ and $\bigcup \partial A \times B ;\{I(r) \times N\}$ from all $i$-flow cells are mutually disjoint. Take $A_{0} \subset A \subset U, A_{0}$ and $A$ compact, $A_{0} \subset A$ is a neighborhood such that $\bar{b} \subset A_{0} \cup N$ is a neighborhood and $I(r) \times A \subset R$. Apply 7.5.1 to change the metric on $I(r) \times U$ to a product metric. Apply 7.5.2 to shrink $I(r) \times U$ to $I \times U$.

$$
I(r)-I\left(r-l \frac{1}{200 n} \frac{1}{8^{i-1}} \cdot \frac{1}{2}\right)
$$

becomes

$$
\begin{aligned}
& I-I\left(1-\frac{l}{r} \cdot \frac{1}{200 n} \cdot \frac{1}{8^{i-1}} \cdot \frac{1}{2}\right), \\
& I-I\left(1-\frac{l}{r} \cdot \frac{1}{200 n} \cdot \frac{1}{8^{i-1}} \cdot \frac{1}{2}\right) \supset I-I\left(1-\left[\frac{l}{r}\right] \cdot \frac{1}{200 n} \cdot \frac{1}{8^{i-1}} \cdot \frac{1}{2}\right) .
\end{aligned}
$$

Note that $[l / r]$ is an integer between 8 and $100 n$. Apply 7.5.4 in whose statement substitute $I(1 / 2)$ by

$$
I\left(1-\left[\frac{l}{r}\right] \cdot \frac{1}{200 n} \cdot \frac{1}{8^{i-1}} \cdot \frac{1}{2}\right)
$$

and $I(3 / 4)$ by

$$
I\left(1-\left[\frac{l}{r}\right] \cdot \frac{1}{200 n} \cdot \frac{1}{8^{i-1}} \cdot \frac{1}{2} \frac{1}{2}\right),
$$

to get a product structure over $I \times\left(A_{0} \cup N\right)$. Apply 7.5.2 to expand $I \times U$ back to $I(r) \times U$.

$$
I-I\left(1-\left[\frac{l}{r}\right] \cdot \frac{1}{200 n} \cdot \frac{1}{8^{i-1}} \cdot \frac{1}{2} \frac{1}{2}\right)
$$

becomes

$$
I(r)-I\left(r-r\left[\frac{l}{r}\right] \cdot \frac{1}{200 n} \cdot \frac{1}{8^{i-1}} \cdot \frac{1}{2} \cdot \frac{1}{2}\right) .
$$

Note that

$$
r\left[\frac{l}{r}\right] \cdot \frac{1}{200 n} \cdot \frac{1}{8^{i-1}} \cdot \frac{1}{2} \frac{1}{2} \geq l \cdot \frac{1}{200 n} \cdot \frac{1}{8^{i}} .
$$

Finally apply 7.5 .1 to change the metric on $I(r) \times U$ back to that on $M$. We have product structure near

$$
\bigcup I\left(l \cdot \frac{1}{200 n} \cdot \frac{1}{8^{i}}\right) \times B \bigcup_{\operatorname{dim} \leq i} a \times b .
$$


In the end we get a product structure near $X$. Changes of control data during the construction are clear. This completes the proof.

\section{Changing $h$-COBordisMS}

8.1. Let $M$ be space with $K \leq 0, \alpha(t)$ curve in $M, \gamma(s)$ geodesic ray such that $\alpha(0)=\gamma(0)$. Consider universal covering $X \rightarrow X / \Gamma=M$. Lift $\alpha(t)$ and $\gamma(s)$ to $\tilde{\alpha}(t)$ and $\tilde{\gamma}(s)$ such that $\tilde{\alpha}(0)=\tilde{\gamma}(0)$. At $\tilde{\alpha}(t)$, draw the geodesic ray asymptotic to $\tilde{\gamma}(s)$. Express the result as $\tilde{\alpha}(t) * \tilde{\gamma}(s)$. For any two liftings the results are the same up to an isometry in $\Gamma$. So we can go back to $M$ to get only one result, denoted $\alpha(t) * \gamma(s)$.

Let $W$ be $h$-cobordism over $M, W \stackrel{p_{t}}{\rightarrow} W$ and $W \stackrel{q_{t}}{\rightarrow} W$ be strong deformations of $W$ to $M$ and to the other boundary, $p=p_{1}: W \rightarrow M$. Consider

$$
\widehat{W}=R(M) X_{M} W=\{(\gamma, x): \gamma \in R(M), x \in W, \gamma(0)=p(x)\} .
$$

It is a manifold with two boundaries, one called $R(M)$. Take a point in $\widehat{W}$, that is, a ray $\gamma$ in $M$ and a point $x \in W$ such that $\gamma(0)=p(x)$. We produce the following two curves in $\widehat{W}$.

$$
\begin{array}{ll}
\left(p\left(p_{t} x\right) * \gamma, p_{t} x\right), & 0 \leq t \leq 1, \\
\left(p\left(q_{t} x\right) * \gamma, q_{t} x\right), & 0 \leq t \leq 1 .
\end{array}
$$

All curves of the first kind form a strong deformation of $\widehat{W}$ to $R(M)$ and those of the second kind form one to the other boundary of $\widehat{W}$. And everything is trivial outside a compact set of $R(M)$. So $\widehat{W}$ is $h$-cobordism over $R(M)$, called the asymptotic lifting of $W$.

8.1.1. Corollary. If $\alpha(t), 0 \leq t \leq 1$, is an associated curve of $W$, then $\{\alpha(t) *$ $\gamma, 0 \leq t \leq 1: \gamma \in R(M), \gamma(0)=\alpha(0)\}$ are associated curves of $\widehat{W}$. If we go through all associated curves of $W$, we get all associated curves of $\widehat{W}$.

8.2. Suppose $X$ is a Hadamard space, $x \in X$ and $z \in \partial X$, then denote the geodesic ray from $x$ to $z$ as $\gamma(x, z)$.

8.2.1. Proposition. Suppose $X$ is a Hadamard space whose geodesic flow is weakly Anosov in the sense of 3.7. $\alpha(t), 0 \leq t \leq 1$, is a continuous curve in $X$.

Denote by $\{\hat{\alpha}(t)\}$ the collection of curves, not necessarily continuous, in $G(X)$ such that $\left.\hat{\alpha}(t)\right|_{[0,+\infty)}=\gamma(\alpha(t), z)$ for some $z \in \partial X$. Then $\{\tau \cdot \hat{\alpha}(t)\}$ has diameter $\leq(l, \varepsilon)$, where $l$ is a constant and $\lim _{r \rightarrow+\infty} \varepsilon=0$.

Proof. Let $u$ be geodesic from $\alpha(0)$ to $z \in \partial X$, and $v$ be geodesic from $\alpha(t)$ to $z$. Inspired by 3.6, we compare $\tau \cdot v$ and $(\tau-B) \cdot u, B=B(u, \alpha(t))$.

Note that the Busemann function depends only on rays. Consider

$$
B(u, \alpha(t))=B(\gamma(\alpha(0), z), \alpha(t)), \quad 0 \leq t \leq 1 .
$$

Let $a=a(z)$ and $b=b(z)$ be its maximum and minimum, then $(\tau-B) \cdot u$ is inside the interval $I=[\tau-a, \tau-b] \cdot u$. By 3.1, length $(I)=2(a-b)$. Let $l$ be the maximum of $2[a(z)-b(z)], z \in \partial X$. 
On the other hand,

$$
\begin{aligned}
& d(\tau \cdot v,(\tau-B) u)=\int_{-\infty}^{+\infty} d(v(x+\tau), u(x+\tau-B)) \cdot e^{-|x|} d x . \\
& \int_{-\infty}^{-\tau+|B|} d(v(x+\tau), u(x+\tau-B)) \cdot e^{-|x|} d x \\
& \quad=\int_{-\infty}^{-\tau+|B|} d(v(x+\tau), u(x+\tau-B)) \cdot e^{x} d x \text { when } \tau \geq|B| \\
& =e^{-\tau+|B|} \cdot \int_{-\infty}^{0} d(v(x+|B|), u(x+|B|-B)) \cdot e^{x} d x \\
& \leq e^{-\tau} \cdot e^{|B|}(d[v(|B|), u(|B|-B)]+2) .
\end{aligned}
$$

Note that the second part of the above expression depends only on $t \in[0,1]$, and $z \in \partial X$ and is continuous. Denote its maximum as $C$.

Define

$$
d(x)=\left\{\begin{array}{lc}
d[v(x), u(x-B)], & x \geq|B|, \\
d[v(|B|), u(|B|-B)], & x \leq|B| .
\end{array}\right.
$$

Note that this function depends only on $x \in \mathbb{R}, t \in[0,1]$ and $z \in \partial X$. It is continuous and bounded. By 3.6, with respect to $x, d$ monotonically decreases to zero.

$$
\begin{aligned}
& \int_{-\tau+|B|}^{+\infty} d(v(x+\tau), u(x+\tau-B)) \cdot e^{-|x|} d x \\
& \quad=\int_{-\tau+|B|}^{+\infty} d(x+\tau) \cdot e^{-|x|} d x \leq \int_{-\infty}^{+\infty} d(x+\tau) \cdot e^{-|x|} d x=f .
\end{aligned}
$$

$\mathbb{R} \times[0,1] \times \partial X \stackrel{f(\tau, t, z)}{\longrightarrow} \mathbb{R} . f$ is continuous and, with respect to $\tau$, monotonically decreases to zero. Denote $m(\tau)=\max \{f(\tau, t, z), t \in[0,1], z \in \partial X\}$. By the following lemma whose proof is left for the reader, $\lim _{\tau \rightarrow+\infty} m(\tau)=0$. Let $\varepsilon(\tau)=e^{-\tau} \cdot C+m(\tau)$. This completes the proof.

8.2.2. Lemma. Suppose $Y$ is a compact topological space, $\mathbb{R} \times Y \stackrel{f(\tau, y)}{\longrightarrow} \mathbb{R}$ is a continuous map, with respect to $\tau, f$ monotonically decreases to zero. $m(\tau)=$ $\max \{f(\tau, y), y \in Y\}$. Then $\lim _{\tau \rightarrow+\infty} m(\tau)=0$.

The following result is proved in the same way, plus 3.4 and 8.1 .

8.2.3. Proposition. Suppose $M$ is a space with $K \leq 0$ whose geodesic flow is weakly Anosov. $P$ is a compact topological space, and $[0,1] \times P \stackrel{\alpha(t, p)}{\longrightarrow} M$ is a continuous map. Denote by $\{\hat{\alpha}(t)\}$ the collection of curves in $G(M)$ such that $\left.\hat{\alpha}(t)\right|_{[0,+\infty)}=\alpha(t, p) * \gamma$ for some $p \in P, \gamma \in R(M), \alpha(0, p)=\gamma(0)$. Then $\{\tau \cdot \hat{\alpha}(t)\}$ has diameter $\leq(l, \varepsilon)$, where $l$ is a constant and $\lim _{\tau \rightarrow+\infty} \varepsilon=0$.

8.2.4. Proposition. Let $M$ be a space with $K \leq 0$ and with condition 4.3, $G(M) \stackrel{f}{\rightarrow} R(M)$ the canonical map. Then for any $\varepsilon>0$ there are $\tau \geq 0$, $\lim _{\varepsilon \rightarrow 0} \tau=+\infty$ and homeomorphism $G(M) \stackrel{g}{\rightarrow} R(M)$ such that $d(\tau \alpha, \tau \beta) \leq \varepsilon$ for any $\alpha, \beta \in G(M), f \alpha=g \beta$.

Proof. Let $X \rightarrow X / \Gamma=M$ be a universal covering. We have $G(X) \stackrel{\pi}{\rightarrow} G(M)$ and $R(X) \stackrel{\pi}{\rightarrow} R(M)$. Fix $\tau \geq 0$. It will be determined. Let us impose the 
following metric on $R(X)$, which generates the correct topology:

$$
d(\alpha, \beta)=d[\alpha(0), \beta(0)]+\int_{-\tau}^{+\infty} d[\alpha(t+\tau), \beta(t+\tau)] \cdot e^{-|t|} d t .
$$

This is $\Gamma$-invariant and we get a metric on $R(M)$ (see [Hu, 5.6]). Apply Theorem 4.4 and take homeomorphism $G(M) \stackrel{g}{\rightarrow} R(M)$ which is $\varepsilon / 2$-close to $f$. Take any $\alpha, \beta \in G(M), f \alpha=g \beta$. Consider $\alpha_{+}=f \alpha=g \beta, \beta_{+}=f \beta$, then $d\left(\alpha_{+}, \beta_{+}\right) \leq \varepsilon / 2$. Take $\hat{\alpha}_{+}, \hat{\beta}_{+} \in R(X)$ such that $d\left(\alpha_{+}, \beta_{+}\right)=d\left(\hat{\alpha}_{+}, \hat{\beta}_{+}\right)$. Extend $\hat{\alpha}_{+}, \hat{\beta}_{+}$to $\hat{\alpha}, \hat{\beta}$ in $G(X)$ such that $\pi \hat{\alpha}=\alpha, \pi \hat{\beta}=\beta, \pi \tau \hat{\alpha}=\tau \pi \hat{\alpha}=$ $\tau \alpha, \pi \tau \hat{\beta}=\tau \beta$. So $d(\tau \alpha, \tau \beta) \leq d(\tau \hat{\alpha}, \tau \hat{\beta}), d\left(\hat{\alpha}_{+}, \hat{\beta}_{+}\right) \leq \varepsilon / 2$ :

$$
\begin{aligned}
& d[\hat{\alpha}(0), \hat{\beta}(0)]+\int_{-\tau}^{+\infty} d[\hat{\alpha}(t+\tau), \hat{\beta}(t+\tau)] \cdot e^{-|t|} d t \leq \varepsilon / 2 . \\
& d(\tau \alpha, \tau \beta) \leq d(\tau \hat{\alpha}, \tau \hat{\beta})=\int_{-\infty}^{+\infty} d[\hat{\alpha}(t+\tau), \hat{\beta}(t+\tau)] \cdot e^{-|t|} d t \\
& \int_{-\tau}^{+\infty} d[\hat{\alpha}(t+\tau), \hat{\beta}(t+\tau)] \cdot e^{-|t|} d t \leq \varepsilon / 2 . \\
& \int_{-\infty}^{-\tau} d[\hat{\alpha}(t+\tau), \hat{\beta}(t+\tau)] \cdot e^{-|t|} d t \\
& \quad \leq \int_{-\infty}^{-\tau}(d[\hat{\alpha}(0), \hat{\beta}(0)]+2|t|) \cdot e^{-|t|} d t \\
& \quad \leq(\varepsilon / 2+2 \tau+2) e^{-\tau} .
\end{aligned}
$$

Pick $\tau=\tau(\varepsilon)$ such that $\varepsilon / 2+(\varepsilon / 2+2 \tau+2) e^{-\tau} \leq \varepsilon, \lim _{\varepsilon \rightarrow 0} \tau=+\infty$. This completes the proof.

8.3. We now proceed to prove the following result. Let $M^{n}$ be a compact space with $K \leq 0$ and with condition 4.3 such that there is no totally geodesic $\mathbb{R} \times[a, b]$ immersed in it. Then $\mathrm{Wh}\left(\pi_{1} M\right)=0$.

By 2.3.2, there is a twisted product $M \times \mathbb{R}$. Take an $h$-cobordism $W$ over $M \times \mathbb{R}$. By 8.1, we have $h$-cobordism $\widehat{W}$ over $R(M \times \mathbb{R}) . \widehat{W}$ is a lifting of $W$. If $\widehat{W}$ is a product, then $\chi\left(S^{n}\right) \cdot \tau(W)=0, \chi\left(S^{n}\right)=1+(-1)^{n}$. Using $\mathbb{R} \times \mathbb{R}=\mathbb{H}^{2}, \partial \mathbb{H}^{2}=\partial^{+} \cup \partial^{0} \cup \partial^{-}$, we have decompositions

$$
G(M \times \mathbb{R})=G=G^{+} \cup G^{0} \cup G^{-}
$$

and

$$
R(M \times \mathbb{R})=R=R^{+} \cup R^{0} \cup R^{-} .
$$

See [FJ1, §2], and [Hu, 6.3]. Clearly, $\widehat{W}=\widehat{W}^{+} \cup \widehat{W}^{0} \cup \widehat{W}^{-}, \widehat{W}^{+} \cup \widehat{W}^{0}$ is an $h$-cobordism over $R^{+} \cup R^{0}$, a fiber bundle over $M \times \mathbb{R}$ with fiber $E^{n+1}$. If $\widehat{W}^{+} \cup \widehat{W}^{0}$ is a product, $\tau(W)=0$.

So consider $\widehat{W}^{+} \cup \widehat{W}^{0}$. By 3.8, $G$ is weakly Anosov. Results 8.1.1, 8.2.3 and 8.2.4 together imply that there is $l>0$ such that for any $\varepsilon>0$ there are $\tau \geq 0$ and homeomorphism $G^{+} \cup G^{0} \stackrel{g}{\rightarrow} R^{+} \cup R^{0}$ such that the $h$-cobordism $\tau g^{-1}\left(\widehat{W}^{+} \cup \widehat{W}^{0}\right)$ over $G^{+} \cup G^{0}$ has diameter $\leq(l, \varepsilon)$. By [FJ1, Lemma 3.8], we need only consider $h$-cobordism $\widetilde{W}$ over $G$ with diameter $\leq(l, \varepsilon)$, where $l$ is constant and $\varepsilon$ is very small. 
Periodic geodesics in $M \times \mathbb{R}$ are in $M \times O=M$. By 2.3.1, in $M$ the number of periodic geodesics $\left\{S^{1}\right\}$ having periods $\leq L$ is finite. Consider the foliated control result 7.6, let $L=l / \delta(n, 1)$, and we can have product structure over $\left.G\right|_{M \times[-r, r]}-\bigcup S^{1}$ with diameter $\leq(L, 1)$. Extend the product over $\bigcup S^{1}$. In conclusion, $\widetilde{W}$ over $G$ has product over $\left.G\right|_{M \times[-r, r]^{\prime}}$ the diameter of $\widetilde{W}$, and the diameter of the product over $\left.G\right|_{M \times[-r,-1] \cup[1, r]}$ have a bound $C$ which is independent of $r$.

Let $G \stackrel{f}{\rightarrow} R$ be the canonical map, $G \stackrel{\pi}{\rightarrow} M \times \mathbb{R}, R \stackrel{\pi}{\rightarrow} M \times \mathbb{R}$, be projections. Take homeomorphism $G \stackrel{g}{\rightarrow} R$ such that $d(\pi g, \pi) \leq 1$ and that

$\left.\left.g G\right|_{M \times[-r, r]} \supset R\right|_{M \times[-r+1, r-1]^{\prime}},\left.\left.\quad g G\right|_{M \times[-r,-1] \cup[1, r]} \supset R\right|_{M \times[-r+1,-2] \cup[2, r-1]}$

$g(\widetilde{W})$ is $h$-cobordism over $R$ with product over $\left.R\right|_{M \times[-r+1, r-1]}$. Since $2 d(\pi, \pi)-4 \leq d$ by 3.5 , where $\pi$ is $G \stackrel{\pi}{\rightarrow} M \times \mathbb{R}, d(\pi, \pi) \leq \frac{1}{2} d+2$, and since $d(\pi g, \pi) \leq 1, d(\pi g, \pi g) \leq \frac{1}{2} d+4$. So the diameter of $g(\widetilde{W})$, and the diameter of the product over $\left.R\right|_{M \times[-r+1,-2] \cup[2, r-1]}$ are bounded by $\frac{1}{2} C+4$ in $M \times \mathbb{R}$.

So we just consider $h$-cobordism $\bar{W}$ over $R$ with product $P$ over $\left.R\right|_{M \times[-r, r]}$, the diameter of $\bar{W}$, and diameter of $P$ over $\left.R\right|_{M \times\{ \pm r\}}$ measured in $M \times \mathbb{R}$ have a bound $C$ which is independent of $r . C$ and $r$ are not those in the preceding paragraph. Consider for example $\left.R\right|_{M \times[r,+\infty)}$. Suppose $\alpha(t) \times \beta(t)$, $0 \leq t \leq 1$, is a curve in $M \times[r,+\infty)$ with length $L$.

$$
\begin{aligned}
L & =\int_{0}^{1} \sqrt{f^{2}(\beta(t)) \cdot\left|\alpha^{\prime}(t)\right|^{2}+\left|\beta^{\prime}(t)\right|^{2}} d t, \\
f(t) & =\frac{e^{t}+e^{-t}}{2} \geq t, \quad t \geq 0, \\
L & \geq \int_{0}^{1} \sqrt{r^{2} \cdot\left|\alpha^{\prime}(t)\right|^{2}+\left|\beta^{\prime}(t)\right|^{2}} d t \\
& =r \cdot \int_{0}^{1} \sqrt{\left|\alpha^{\prime}(t)\right|^{2}+\left|\frac{\beta^{\prime}(t)}{r}\right|^{2}} d t .
\end{aligned}
$$

Consider $\left(\mathrm{Id} \times \frac{1}{r} \mathrm{Id}\right) \circ \pi:\left.R\right|_{M \times[r,+\infty)} \rightarrow M \times[r,+\infty) \rightarrow M \times[1,+\infty)$. The diameters measured in $M \times[1,+\infty)$, with product metric, have a bound $\frac{1}{r} C$. Let $r$ be large. Also note that (Id $\left.\times \frac{1}{r} \mathrm{Id}\right) \circ \pi$ is a fiber bundle with fiber $S^{n}$. By the ordinary control Theorem 5.1,P can be extended. This completes the proof of 8.3.

It is left for the reader to check that instead of $M$, we can consider $M \times T^{k}$, $k \geq 0$. Then $\mathrm{Wh}\left(\pi_{1} M \times \mathbb{Z}^{k}\right)=0, k \geq 0$. That is the following theorem.

8.4. Theorem. Let $M$ be compact space with $K \leq 0$ and with condition 4.3 such that there is no totally geodesic $\mathbb{R} \times[a, b]$ immersed in it. Then

$$
\mathrm{Wh}\left(\pi_{1} M \times \mathbb{Z}^{k}\right)=0, \quad k \geq 0 .
$$

8.4.1. Corollary. Let $M$ be a compact riemannian or PL manifold with $K \leq 0$ such that there is no totally geodesic $\mathbb{R} \times[a, b]$ immersed in it. Then

$$
\mathrm{Wh}\left(\pi_{1} M \times \mathbb{Z}^{k}\right)=0, \quad k \geq 0 .
$$


8.4.2. Corollary. Let $M$ be a compact PL manifold with $K \leq \varepsilon<0$. Then $\mathrm{Wh}\left(\pi_{1} M \times \mathbb{Z}^{k}\right)=0, k \geq 0$.

\section{Hyperbolization}

This section shall extend result 8.4.2 from manifolds to polyhedra. Actually we shall use Gromov's hyperbolization to see a simple fact: The fundamental group of a finite polyhedron is built up by fundamental groups of closed PL manifolds via free products with amalgamations. Now, concerning Whitehead groups, there is 8.4.2 for manifolds and Waldhausen's result for free products with amalgamations.

9.1. Recall the construction of hyperbolization [G1, 3.4]. Let $K$ be compact simplicial complex with PL flat geometry, and $A$ be subcomplex. Define $h K^{1}=$ $K^{1}$. For any $\Delta^{2} \subset A$, define $h \Delta^{2}=\Delta^{2}$. For any $\Delta^{2} \not \subset A$, there is compact 2-dimensional PL manifolds $h \Delta^{2}$ with $\partial h \Delta^{2}=h \partial \Delta^{2}=\partial \Delta^{2}$. Glue all $h \Delta^{2}$ to $K^{1}$ and define $h K^{2}=K^{1} \cup h \Delta^{2}$. Assume that we already have $h K^{i-1}$. For any $\Delta^{i} \subset A$, define $h \Delta^{i}=\Delta^{i}$. For any $\Delta^{i} \not \subset A$, there is a compact PL manifold $h \Delta^{i}$ with $\partial h \Delta^{i}=h \partial \Delta^{i}$. Glue all $h \Delta^{i}$ to $h K^{i-1}$ and define $h K^{i}=h K^{i-1} \cup h \Delta^{i}$. Finally we have a compact simplicial complex $h K$ with PL flat geometry, called the hyperbolization of $K$ relative to $A$. It is important that for any $\Delta^{i} \not \subset A, \partial h \Delta^{i} \subset h \Delta^{i}$ is totally geodesic and $h \Delta^{i}$ has nonpositive curvature. (One can assume, after a barycentric subdivision, that in $K$ the intersection of any simplex with $A$ is a simplex.)

9.1.1. Remarks. (1) For any subcomplex $L \subset K, h L \subset h K$ is precisely the hyperbolization of $L$ relative to $L \cap A$. (2) If $K$ is PL manifold, then $h K$ is PL manifold.

9.1.2. Lemma. Suppose that $X$ and $Y$ are length spaces, $A$ compact, $A \subset X$, $A \subset Y, X \cup Y=X \uplus_{A} Y=X \uplus Y$ /Identify $A, P \subset X$ and $Q \subset Y$ are totally geodesic, $P \supset A, Q \supset A$. Then $P \cup Q \subset X \cup Y$ is totally geodesic.

The proof is left to the reader.

9.1.3. Lemma. Let $P \subset Q$ be subcomplexes in $K$. Then $A \cup h P \subset A \cup h Q$ is totally geodesic.

Proof. It is enough to show that for any subcomplex $L$ and any simplex $\Delta^{i}$ with $\partial \Delta^{i} \subset L, A \cup h L \subset A \cup h\left(L \cup \Delta^{i}\right)$ is totally geodesic. For $\Delta^{i} \subset A$, there is nothing to prove. So assume that $\Delta^{i} \not \subset A$.

Note that $A \cap h\left(L \cup \Delta^{i}\right)=A \cap h L$. In fact

$$
A \cap h \Delta^{i}=A \cap \Delta^{i}=A \cap \partial \Delta^{i}=A \cap h \partial \Delta^{i} \subset h \partial \Delta^{i} \subset h L .
$$

So, by 9.1.2, we just prove $h L \subset h\left(L \cup \Delta^{i}\right)=h L \cup h \Delta^{i}$ to be totally geodesic. As $h L \cap h \Delta^{i}=h\left(L \cap \Delta^{i}\right)=h \partial \Delta^{i}, h \partial \Delta^{i}=\partial h \Delta^{i} \subset h \Delta^{i}$ is totally geodesic, $h L \subset h L \cup h \Delta^{i}$ is totally geodesic by 9.1.2.

9.1.4. Lemma. For any subcomplex $L$ and simplex $\Delta^{i}, \partial \Delta^{i} \subset L, \Delta^{i} \not \subset A$, $h \partial \Delta^{i} \subset A \cup h L$ is totally geodesic.

Proof. $A \cap h \partial \Delta^{i}=A \cap \partial \Delta^{i}=A \cap \Delta^{i} . A \cap \Delta^{i} \subset A$ is totally geodesic. By 9.1.2, $h \partial \Delta^{i} \subset A \cup h \partial \Delta^{i}$ is totally geodesic. But $A \cup h \partial \Delta^{i} \subset A \cup h L$ is totally geodesic by 9.1 .3 . 
9.1.5. Remarks. (1) Since [G1, 4.3] one can glue spaces with curvature $\leq 0$ along compact totally geodesic subsets and still have curv. $\leq 0$, one sees that when $A$ has curv. $\leq 0, h K$ has curv. $\leq 0$. (2) When $A$ has curv. $<0$, i.e., any link of $A$ is strictly larger than a standard sphere, then $h K$ can have curv. $<0$ after a very very subtle perturb, provided a good hyperbolization is in use. See [G1, 3.4, 3.4C and 4.3A] . (3) When $A$ is aspherical, $h K$ is aspherical [G1, 3.4C].

9.1.6. Lemma. Let $X$ be a space with $K \leq 0, A \subset X$ be compact totally geodesic. Then $\pi_{1} A \rightarrow \pi_{1} X$ is injective.

Proof. The familiar Hadamard trick indicates that any nontrivial element in $\pi_{1} A$ is realized as a periodic geodesic $\alpha . \alpha$ in $X$ is periodic geodesic. Since $X$ has $K \leq 0, \alpha$ is not homotopic to a point.

9.1.7. Recall that for groups $G$ and $\widetilde{G}$ and subgroup $H \subset G, H \subset \widetilde{G}$, there is a free product with amalgamation $G *_{H} \widetilde{G}=G * \widetilde{G} /$ Identify $H$. Also note that $\left(G *_{H} \widetilde{G}\right) \times L=(G \times L) *_{H \times L}(\widetilde{G} \times L)$.

Reconsider the construction of $h K$. Corresponding fundamental groups are as follows:

$$
\begin{aligned}
& A \rightarrow \pi_{1} A \\
& A \cup K^{1} \rightarrow \pi_{1} A * \mathbb{Z} * \cdots * \mathbb{Z} \\
& \quad \vdots \\
& \quad A \cup h L \rightarrow \pi \\
& \quad \Delta^{i} \not \subset A, \Delta^{i} \not \subset L, \partial \Delta^{i} \subset L, A \cup h L \cup h \Delta^{i} \rightarrow \pi *_{\pi_{1} h \partial \Delta^{i}} \pi_{1} h \Delta^{i} \\
& \quad \vdots \\
& h K \rightarrow \pi_{1} h K
\end{aligned}
$$

9.2. Theorem (F. Waldhausen [W1, p. 157]).

$$
\mathrm{Wh}(H) \rightarrow \mathrm{Wh}(G) \oplus \mathrm{Wh}(\widetilde{G}) \rightarrow \mathrm{Wh}\left(G *_{H} \tilde{G}\right)
$$

is exact.

9.3. Theorem. Let $K$ be a compact simplicial complex with curvature $<0$. Then $\mathrm{Wh}\left(\pi_{1} K \times \mathbb{Z}^{k}\right)=0, k \geq 0$.

Proof. After a subdivision, we may assume that $K$ is a subcomplex of compact PL manifold $M$. For any simplex $\Delta^{i} \not \subset K, \partial \Delta^{i} \cap K=\Delta^{i} \cap K, \Delta^{i} \cap K \subset K$ is totally geodesic and hence has curv. $<0$. Since $\partial \Delta^{i}$ is PL manifold, $h \partial \Delta^{i}$ is PL manifold. So $h \partial \Delta^{i}$ is a compact PL manifold with curv. $<0$. So, by 8.4.2, $\mathrm{Wh}\left(\pi_{1} h \partial \Delta^{i} \times \mathbb{Z}^{k}\right)=0, k \geq 0$. Consider the fundamental group situation of hyperbolization and Waldhausen's result. We see that $\mathrm{Wh}\left(\pi_{1} K \times \mathbb{Z}^{k}\right) \subset$ $\mathrm{Wh}\left(\pi_{1} h M \times \mathbb{Z}^{k}\right), k \geq 0, h M$ is compact PL manifold with curv. $<0$. By 8.4.2, $\mathrm{Wh}\left(\pi_{1} h M \times \mathbb{Z}^{k}\right)=0, k \geq 0$. So $\mathrm{Wh}\left(\pi_{1} K \times \mathbb{Z}^{k}\right)=0, k \geq 0$.

9.3.1. Remark. In the same way, the following first conjecture implies the second. Compare [Hs, p. 114].

Conjecture 1. For any aspherical compact PL manifold $M$, Wh $\pi_{1} M=0$.

Conjecture 2. For any compact aspherical simplicial complex $K$, Wh $\pi_{1} K$ $=0$. Tom Farrell mentioned that a similar remark on the Novikov conjecture was made by Davis in [D, p. 215]. (As for 9.1.7, we have two explicit constructions available for the case of nonpositive curvature.) 
9.4. Some direct applications. (1) Let $K$ be any finite polyhedron. Hyperbolize the whole $K: K \rightarrow h K$. Then $\mathrm{Wh}\left(\pi_{1} h K \times \mathbb{Z}^{i}\right)=0, i \geq 0$.

(2) Let $\Gamma$ be a finitely presented group. Then $\Gamma=\pi_{1} K$, where $K$ is a compact 2-simplicial complex. We have corresponding expression

$$
\Gamma=\left\{a_{1}, \ldots, a_{k} ; w_{1}=e, \ldots, w_{l}=e\right\} .
$$

Now substitute each 2-simplex of $K$ by a Möbius strip: $K \rightarrow \widetilde{K}, \widetilde{K}$ can be a compact 2 -simplicial complex with curvature $<0$. Its fundamental group is

$$
\widetilde{\Gamma}=\left\{a_{1}, \ldots, a_{k}, b_{1}, \ldots, b_{l} ; w_{1}=b_{1}^{2}, \ldots, w_{l}=b_{l}^{2}\right\} .
$$

So $\operatorname{Wh}\left(\widetilde{\Gamma} \times \mathbb{Z}^{i}\right)=0, i \geq 0$. This construction is from [G1, 3.4]. There are various variations. For example, one can use some punctured tori, or one can leave one or two 2-simplexes unchanged.

(3) Consider Coxeter groups. Consider

$$
G=\left\{a_{1}, \ldots, a_{k} ;\left(a_{i} a_{j}\right)^{d_{i j}}=e\right\} .
$$

Assume that $d_{i i}=1, d_{i j} \neq 1, i \neq j$. Also assume that $G$ is infinite and contains no $\mathbb{Z} \oplus \mathbb{Z}$. G. Moussong in [Mo] showed that $G$ acts on a simply connected polyhedron $X$ with $K<0$ and is cocompact. $G$ has a torsion-free cofinite subgroup $\Gamma$, by Selberg's lemma. Then $X / \Gamma$ is compact with $K<0$. So $\mathrm{Wh}\left(\Gamma \times \mathbb{Z}^{i}\right)=0, i \geq 0$. Also see [G1, 3.4.D].

\section{REFERENCES}

[B] M. Brown, Some applications of an approximation theorem for inverse limits, Proc. Amer. Math. Soc. 11 (1960), 478-481.

[CF] T. A. Chapman and S. Ferry, Approximating homotopy equivalences by homeomorphisms, Amer. J. Math. 101 (1979), 583-607.

[D] M. Davis, Coxeter groups and aspherical manifolds, Lecture Notes in Math., vol. 1051, Springer, 1984, pp. 197-221.

[DJ] M. W. Davis and T. Januszkiewicz, Hyperbolization of polyhedra, J. Differential Geom. (to appear).

[EK] R. D. Edwards and R. C. Kirby, Deformations of spaces of imbeddings, Ann. of Math. 93 (1971), 63-88.

[EO] P. Eberlein and B. O'Neill, Visibility manifolds, Pacific J. Math. 46 (1973), 45-110.

[FJ1] F. T. Farrell and L. E. Jones, K-theory and dynamics. I, Ann. of Math. 124 (1986), 531-569.

[FJ2] _ K K-theory and dynamics. II, Ann. of Math. 126 (1987), 451-493.

[FJ3] _ Rigidity and other topological aspects of compact nonpositively curved manifolds, Bull. Amer. Math. Soc. 22 (1990), 59-64.

[G1] M. Gromov, Hyperbolic groups, Essays in Group Theory (S. M. Gersten, ed.), MSRI Publ. 8 (1987), 75-263.

[G2] _ Hyperbolic manifolds, groups and actions, Ann. of Math. Studies, no. 97, Princeton Univ. Press, 1981, pp. 183-351.

[G3] _- Infinite groups as geometric objects, Proc. ICM Warsaw, Vol. 1, 1984, pp. 385-391.

[Hs] W. C. Hsiang, Geometric applications of algebraic K-theory, Proc. ICM, Warsaw, Vol. 1, 1983, pp. 99-118.

[Hu] B. Hu, h-cobordisms over certain nonpositively curved spaces, Thesis, SUNY, Stony Brook, 1989.

[Ks] R. C. Kirby and L. C. Siebenmann, Foundational essays on topological manifolds, Smoothings and Triangulations, Ann. of Math. Studies, no. 88, Princeton Univ. Press, 1977. 
[Mi] J. Milnor, Microbundles, Part I, Topology 3 (1964), Supplement 1, 53-80.

[MO] G. Moussong, Hyperbolic coxeter groups, Thesis, Ohio State Univ., 1988.

[Q1] F. Quinn, Ends of maps. I, Ann. of Math. 110 (1979), 275-331.

[Q2] _ Topological transversality holds in all dimensions, Bull. Amer. Math. Soc. 18 (1988), 145-148.

[Se] A. Selberg, On discontinuous groups in higher dimensional symmetric spaces, Internat. Colloq. on Function Theory, Tata Inst., Bombay, 1960.

[Si] L. C. Siebenmann, Approximating cellular maps by homeomorphisms, Topology 11 (1973), 271-294.

[T] J. L. Tits, On buildings and their applications, Proc. ICM Vancouver, 1974, pp. 209-221.

[W1] F. Waldhausen, Whitehead groups of generalized free products, Lecture Notes in Math., vol. 342, 1973, Springer, pp. 155-179.

[W2] __ Algebraic K-theory of generalized free products, Ann. of Math. 108 (1978), 135-256.

Chinese Academy of Sciences, Quanzhou Health School, Fujian, China

IHES, 91440 Bures-SUR-YvetTe, France

Current address: Max-Planck-Institute für Mathematik, 5300 Bonn 3, Germany

E-mail address: bizhong@eurydice.mpim-bonn.mpg.de 